\title{
Structured Microgels through Microfluidic Assembly and Their Biomedical Applications
}

\author{
Rong-Cong Luo, Chia-Hung Chen ${ }^{*}$ \\ Department of Bioengineering, National University of Singapore, Singapore City, Singapore \\ Email: biecch@nus.edu.sg
}

Received October 10, 2012; revised November 19, 2012; accepted December 7, 2012

\begin{abstract}
Droplet-based microfluidic is an effective, versatile and scalable approach which can be used to produce structured microgels with desirable features. The high degree of control endowed with microfluidics enables the formation of various functional microgels such as multi-compartment encapsulations, Janus-shaped particles and non-spherical microgels. These microgels have aroused great interest in biological engineering aspect, since they outperform their counterparts produced from other techniques and have been applied in drug delivery, 3-Dimensional cell culture, micro-tissues, single cell assay, tissue engineering and bio-imaging. In this review, we will summarize the fabrication processes, technology comparisons and the usages in biomedical applications.
\end{abstract}

Keywords: Droplet Microfluidic; Microgels; Cell Encapsulation; Tissue Engineering

\section{Introduction}

Microgels are colloidal gel particles with dimensions in the range from tens to hundreds of micrometers, which are consisted with cross-linked three-dimensional polymeric network [1]. One of the most important characteristics for microgels is their swelling to adsorb and to hold large amounts of aqueous solvents in response to environmental stimuli such as $\mathrm{pH}$, temperature, ionic strength, electric and magnetic fields [2]. Such porous aqueous microenvironment created by microgels is quite useful and beneficial for the encapsulation of bioactive substances including peptides, proteins, enzymes, DNA, drugs and cells, since it provides mimetic in vivo aqueous environment where peptides or enzymes are prevented from drying [3]. Furthermore, polymer materials for making microgels can be modified to be stimuli-responsive. Stimuli-responsive microgels enable the drug release in an on-demanded manner with precise concentration, which is quite desirable in advanced drug delivery [4]. Consequently, microgels have been seen a dramatic rise in therapeutic agents encapsulation and delivery.

Traditionally, microgels are prepared with high-shear emulsified techniques such as precipitation, spray-drying and phase separation. Microgels produced from these techniques have large size dispersity, high variability of uncontrollable structures and limited encapsulation efficiency $[5,6]$. Recently, the microfluidic technology offered a method to shape and assemble the microgels

"Corresponding author. through physical shear forces without relying on chemical synthesis, which largely widen the applications of microgels in biomedical fields. A number of reviews and research papers have given overall progress, versatility and superiority of producing microgels with droplet microfluidic techniques over the conventional methods [7-11]. These microgels open up applications in drug delivery, cell encapsulation, bio-sensing and tissue engineering $[12,13]$. The mono-dispersed emulsions made through microfluidics were produced with well-defined size and shape [12] for enhanced control of drug delivery kinetic, which is important for many applications. For example, Berkland et al. showed that $10 \mu \mathrm{m}$ poly (lactic acid-co-glycolic acid) (PLGA) microparticles exhibited a concave downward shape typical diffusion-controlled release while profiles from 50 and $100 \mu \mathrm{m}$ particles showed a sigmoidal shape. Furthermore, combination of different sized of microparticles can produce zero-order release profiles which are quite desirable for drug delivery [14-16].

The structured microgels with well defined morphology such as Janus particles, multi-compartment microgels and non-spherical microgels can be conveniently produced with droplet microfluidic, which is quite difficult for bulk emulsion methods $[17,18]$. Geometry of the microgels has been demonstrated to have an important impact on the biological processes, such as vasculature, circulation time, targeting efficiency phagocytosis, endocytosis and subsequent intracellular transport for therapeutic delivety [19]. The high degree of control af- 
forded by droplet microfluidic enables generation of single and multiple emulsion droplets, which can be transformed to non-spherical microparticles through further simple processing steps.

In this review, we discuss the fabrications of microgels through droplet based microfluidic and their biomedical applications. We summarize and compare different methods such as photo-initiated chemical crosslinking and physically induced gelation of droplets based on their technical specifications, beneficial features and disadvantages. The recent works from different groups are highlighted which use various microfluidic strategies to prepare controlled structural microgels. The methods to fabricate structured microgel with precise control of the size, shape, and encapsulating agents through microfluidics are summarized. In addition, the review also illustrates the biomedical applications of microgels generated, specifically focusing on drug delivery, cell encapsulation and tissue engineering.

\section{Production of Droplets}

Microfluidic production of microgels broadly consists of two steps. In the first step, droplets of solution of pre-gel polymers or monomers are produced in microfluidic device via emulsification of the pre-gel solution with the oil phase. Pre-gel solution phase (i.e. water phase) has been formed with various materials. For example, chemical gelation has been exploited for the microfluidic production of microgels by using photo-initiated or redox-initiated polymerization of $\mathrm{N}$-isopropylacrylamide, dextranhydroxymethyl methacrylate (dex-HEMA), acrylamide and poly(ethylene glycol) diacrylate (PEG-DA) [20-22]. Physical gelation is also used for preparing microgels from natural polymers such as agarose, alginate, carrageenan, chitosan, gelatin, carboxymethylcellulose (CMC) and pectin. Currently, cells, enzyme, peptides, DNA, small molecular drugs, virus and various nanoparticles have been incorporated in the water phase to produce microgels with versatile properties [23-25]. The key requirement for the pre-gel materials and encapsulating agents is their immiscibility with the continuous oil phase, which can ensure all encapsulating agents are contained inside the droplets and formation of stable droplets without aggregations [26,27].

For the continuous oil phase, mineral oil, silicone oil, corn oil, hexadecane and fluorinated oil have been used for generating pre-gel emulsion droplets. Viscosity of the oil phase is one of important factors controlling the droplet generation. Stone and co-workers reported the results of a comparative study of microfluidic emulsion of liquids with different viscosities of oil phase. They reported that highly viscous liquids were emulsified into larger droplets with lower polydispersity. Although it was not possible to provide a unified scaling for the volumes of the results, their results suggested that the breakup dynamics of the lower viscosity fluids resembles the rate-of-flow-controlled bread-up. Their results may be helpful for a rationalized selection of liquids for the controlled formation of droplets with predetermined size and with a narrow distribution of sizes [28,29]. Considering the potential biomedical application of the formed microgels, fluorinated oil has many advantages over conventional hydrocarbon oils, such as improved oxygen permeability and immiscibility with organic compounds, which is quite desirable for cell encapsulation application in microgels [30].

Although droplet formation can be achieved via emulsifying the continuous oil phase and dispersed water phase in the microfluidic channel, droplets will rapidly coalesce inside the devices without surfactants as the stabilizing agents. The surfactants must meet some requirements such as providing stability of the droplets, preventing coalescence and producing biologically inert and biocompatible surface for the water droplets. Typically, hydrocarbon or fluorocarbon oils are used as the oil phase for the w/o emulsions. For the hydrocarbon oil like mineral oils, commercially available surfactants such as Span 80 and AbilEM have been most widely used [31]. For the fluorocarbon oils, surfactants with short fluorotelomer-tails have been used, but unable to provide sufficient long-term emulsion stability. Other fluorosurfactants with longer fluorocarbon tails, such as perfluorinated polyether (PFPE), offer long term stabilization but their ionic headgroups will interact with charged bioactive compounds and cause lysis of cells, which hampers their biomedical application. In order to improve the biocompatibility of the fluorosurfactants, adding hydrophilic head groups like oligoethylene glycol is proved to be a good way [32,33]. Clausell-Tormos et al. investtigated the effects of different hydrophilic head groups on the biocompatibility of the surfactants. They found that surfactants having the ammonium salt of carboxyPFPE and poly-L-lysine as head groups affected lysis of HEK293T in cells, whereas surfactants with polyethyleneglycol (PEG) allowed cultures to develop and proliferate in a similar fashion to control cells grown without surfactant [34].

Combined utilization of fluorinated liquids as the oil phase and fluorosurfactants as the droplets stabilizing agents has been showing promising advantages over the other combination in terms of reducing phase partition, improving gas exchange, improving biocompatibility and enhancing droplets long-term stability. The Novec ${ }^{\mathrm{TM}}$ Engineering Fluid Novec ${ }^{\mathrm{TM}}$ HFE-7500 (3-ethoxy-dodecafluoro-2-trifluoromethyl-hexane) and HFE-7100 (a mixture of methylnonafluorobutyl ether and methyl nonafluoroisobutyl ether) are semi-fluorinated compounds [35], 
which have been frequently used as the continuous oil phases. Regarding the fluorosurfactants, currently, the most promising molecules consist of a block copolymer of PFPE and PEG. The PFPE chosen for the tail (outer block) provides good stabilization of the droplets while the PEG moieties chosen for the headgroup (inner block) prevent adsorption of biological materials. The molecular weight of PEG block is the crucial factor for the performance of these fluorosurfactants. The PEG moiety must be large enough to provide sufficient anchoring strength, but must nonetheless remain slightly smaller than the outer PFPE block to maintain a surfactant geometry that oppose neck formation which can lead to coalescence [36]. The PEG moiety can also be replaced with a similar molecule named Jeffamine ${ }^{(B}$ ED-900 which is PEG-based polyetheramine molecules. Jeffamine ${ }^{\circledR}$ ED900 is more economically competitive than PEG and has some combined benefits such as flexibility from polyether, hydrophilicity from PEG, reactivity from amine end groups and biocompatibility from PEG [37]. In general, the surfactants need meet the requirement of the oil phase used. The details of surfactant-oil combinations are summarized (Table 1) [38].

Droplets can be produced in microfluidic devices with different geometries according to different requirements in liquid viscosity and flow rate controls, such as flowfocusing device, T-junction, co-axial capillaries and micro-nozzle cross-flow system [39-41]. We specifically discussed $\mathrm{T}$-junction and flow-focusing geometries in this section. Regarding the T-junction configuration, the inlet channel with the water dispersed phase perpendicularly intersects the main channel containing the continuous phase. The forces generated by the continuous phase and the subsequent pressure gradient caused the head of the dispersed phase to elongate into the main channel until the neck of the dispersed phase breads the stream into a droplet. Fluid flow rates, channel widths and viscosity of the phases will affect the sizes of the droplets
$[42,43]$. In the flow-focusing configuration, the dispersed and continuous phases are forced through a narrow region in the microfluidic device. The design employs symmetric shearing by the continuous phase on the dispersed phase to generate droplets. Since T-junction droplet makers use two inlets and flow-focusing droplet makers used three inlets and symmetric junction, they will have difference in performance and produce droplets with different properties. Abate et al. presented a direct comparison between T-junction and flow-focusing devices in producing droplets based on studying different capillary numbers (Ca) [44]. By fixing the viscosity and surface tension values, they found that, in T-junction, the asymmetric injection of the fluids leads to a stable droplet formation at low $\mathrm{Ca}$ and jetting at high $\mathrm{Ca}$. In contrast, in flow-focusing devices, the symmetric injection leads to unstable poly-disperse droplet formation at low $\mathrm{Ca}$ and monodisperse droplets formation at high Ca values [44].

\section{Bulky Fabrication}

In order to enhance the throughput of droplets generated from the microfluidic devices, Nakajima and co-workers demonstrated using straight-through micro-channel emulsification as the solution for the high-throughput production of droplets [45] (Figure 1(a)). A straightthrough micro-channel is a channel array microfabricated vertically to the surface of a silicon plate (Figure 1(a)). As Figure 1(a) indicated, the continuous phase was supplied into the module by lifting a tank filled with the continuous phase. The to-be-dispersed phase, infused from the back of the plate, passed through the channels; droplets are then formed in the continuous phase. Channel cross-section and flow rate of oil phase are two important factors affecting the generation of droplets and their size distribution. Oblong channel exceeding a threshold aspect ratio are suitable for forming droplets with less coefficient of variation while use of circular and

Table 1. Surfactants and oils for forming droplets in microfluidics.

\begin{tabular}{|c|c|c|c|c|c|}
\hline \multicolumn{3}{|c|}{ Surfactants } & \multicolumn{3}{|c|}{ Oils } \\
\hline For silicon oils & For hydrocarbon oils & For fluorinated oils & Silicon & Hydrocarbon & Fluorinated \\
\hline $\begin{array}{l}\text { Triton X-100; } \\
\text { SDS(in water); } \\
\text { ABIL }^{\circledR} \text { EM90; }\end{array}$ & $\begin{array}{c}\text { Span 80; } \\
\text { Monolein Oleic acid; } \\
\text { Tween 20/80; } \\
\text { Synperonic PEF; SDS (in } \\
\text { water); } \\
n \text {-Butanol; } \\
\text { ABIL }{ }^{\circledR} \text { EM90; } \\
\text { Phospholipids; }\end{array}$ & $\begin{array}{c}\text { PF-octanol; PF-decanol; } \\
\text { PF-TD acid; } \\
\text { PF-TD OEG; } \\
\text { PFPE-COOH; } \\
\text { PFPE-COONH4; } \\
\text { PFPE-PEG; PFPE-DMP; }\end{array}$ & $\begin{array}{l}\text { DC200; } \\
\text { PDMS; } \\
\text { AR200; }\end{array}$ & $\begin{array}{l}\text { Hexadecane; } \\
\text { Tetradecane; } \\
\text { Octadecane; } \\
\text { Dodecane; Mineral } \\
\text { oil; Isopar M; } \\
\text { Vegetable oil; }\end{array}$ & $\begin{array}{c}\text { PFH; } \\
\text { PFC; } \\
\text { PFD; } \\
\text { PFPH; } \\
\text { HFE (7100, 7200, } \\
\text { 7500); FC (40, } \\
70,77,3283) ;\end{array}$ \\
\hline
\end{tabular}

Notes: Span 80 - is Sorbitane monooleate nonionic surfactant; SDS-Sodium Dodecyl Sulfate; ABIL ${ }^{\circledR}$ EM900-is a nonionic, liquid emulsifier which is based on silicone; Synperonic ${ }^{\mathrm{TM}} \mathrm{PEF}$ - is a polyalkylene oxide block copolymer; PF-perfluoro; TD — tetradecanoic; PFH-perfluoro-hexane; PFC-perfluo-rocyclohexane; PFD - perfluoro-decaline; PFPH - perfluoro-perhydrophenanthree; HFE (7100, 7200, 7500) - ${\operatorname{are~} 3 \mathrm{M}^{\mathrm{TM}} \text { Novec }}^{\mathrm{TM}}$ Engineered fluids; FC (40, 70, 77, 3283 ) - are $3 \mathrm{M}^{\mathrm{TM}}$ Fluorinert ${ }^{\mathrm{TM}}$ Engineered fluids. 


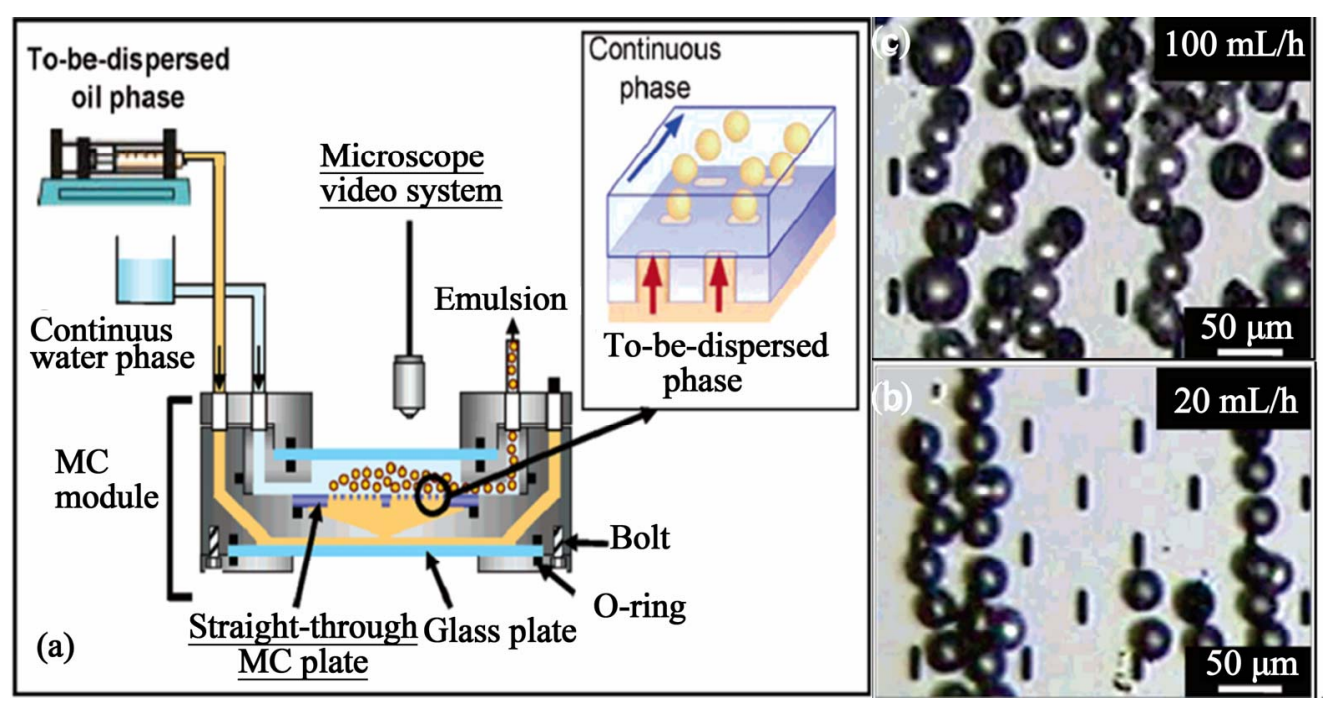

Figure 1. (a) Schematic of the apparatus for the straight-through micro-channel emulsification for large scale generation of droplets; (b) formation of monodisperse; and (c) polydisperse droplets at different flow rates (from ref. 45 with permission (C) 2005, American Chemical Society).

oblong channels below the threshold aspect ratio resulted in continuous outflow of the to-be-dispersed phase that passed through the channels. Meanwhile, critical flow rate of the oil phase is important for the size distribution of the formed droplets. Below the critical flow rate, the oil phase was transformed into monodisperse emulsion droplets (Figure 1(b)) while above the critical flow rate, poly-disperse emulsion droplets are formed (Figure 1(c)). One of the biggest advantages of this method is the high productivity of droplets compared to the single microfluidic device. The devices used in their study have maximum throughput capacities of droplets of $20-30 \mathrm{ml} / \mathrm{h}$ for soybean oil and $60-70 \mathrm{ml} / \mathrm{h}$ for silicon oil. Another beneficial feature of this method is that this platform did not use Polydimethylsiloxane (PDMS) as the materials, which will become swelling when repeated using and is incompatible with organic solvents. Although increased throughput of droplets is achieved, the coefficient of variation of the generated droplets is larger (about 10\%) than those from single microfluidic devices (about 2\%). Consequently, refining the size distribution of droplets from this method may be needed for industrial usage.

In order to generate droplets with submicron size, the same group used a micro-fabricated channel array on a single silicon plate $[46,47]$. They used sub-micron channel arrays with an equivalent diameter as small as 0.57 $\mu \mathrm{m}$ to form oil-in-water emulsions with fine droplets (Figure 2). The sub-micron channels arrays consists of channels and a terrace, with a deep well over the terrace edge. A total of 1500 channels are positioned on the silicon plate. Droplets generation process for these devices is a unique one based on spontaneous transformation of the distorted dispersed phase into spherical droplets. This spontaneous transformation-based droplets generation is

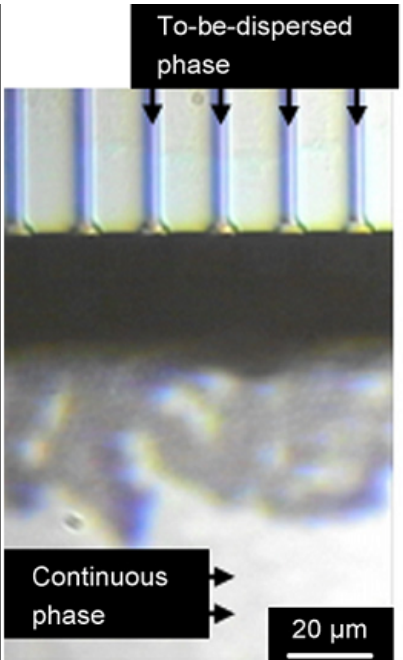

(a)

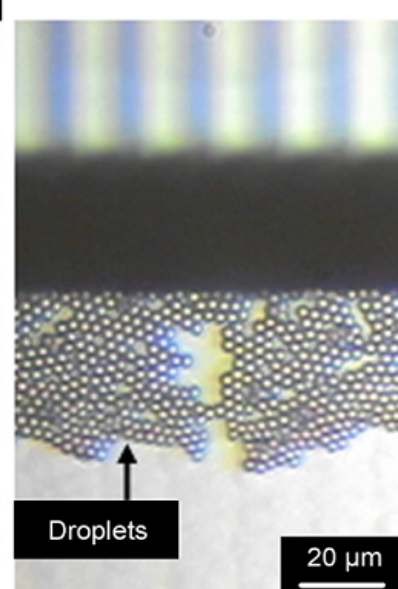

(b)
Figure 2. (a) Microscope image of successful droplets generation from sub-micron channel arrays (b) the droplets generated using the device (from ref. 46 with permission (c) 2007, Elsevier).

driven by instability and non-linearity of the flow of the two phases due to interfacial tension effects on the terrace and in the well. By using the paralleled channels, droplets with diameter of $1.4-3.5 \mu \mathrm{m}$ and minimum coefficients of variation are successfully formulated. The droplet production rate of single silicon plate can reach up to $2.2 \times 10^{4}$ droplets/s. One important feature of this design is that the high throughput production of submicron droplets can be achieved in a minimized single silicon plate. Besides the numbers of droplets, different kinds of droplets containing various reagents may also be simultaneously encapsulated into the droplets using this 
design, which can be a droplets library for droplet-based high throughput multiplexed bioassay application [48].

\section{Gelation of the Droplets}

The formed droplets can be solidified via on-chip or off-chip gelation of the pre-gel solution droplets. On-chip gelation refers to gelling of the droplets during their residence in the microchannels while off-chip gelation means gelling the droplets by collecting them in separated vials where curing agents are presented [49-51]. On-chip gelation may need introduction of extra channels such as side channels for feeding of curing agents and spiral channel or individual compartments for the reaction of curing agents with the gel materials, which may increase flow resistance of the devices. Meanwhile, modification of PDMS channel, such as mixing PDMS elastomer with fluorescent dapoxy dyes to reduce UV light scattering, can enhance the UV on-chip gelation efficiency. This method will need synthesis of special silica beads and may cause perturbation of the steady flow state after long time UV exposure [52]. Another challenge for on-chip gelation is the easy blocking of the channel due to the leakage of curing agents or the delaying efflux of formed microgels from the channels. Moreover, when the microgels are used for cell encapsulation, on-chip UV gelation will require high intensity UV dosage, longer UV irradiation time and high amounts of photo initiators, which are harmful to the cell viability [53].

On the other hand, off-chip gelation which separates the droplet fabrication and gelation steps can use simpler devices design and can eliminate the problems of channels blocking. However, off-chip gelation may be limited by the inefficiency and possibility of producing polydisperse microgels. Since the produced droplets are fragile and easy to break before solidification, any physical forces like shaking and stirring which is re- quired to have homogenous curing reaction will cause break of the droplets and form coalescence between droplets [54]. Furthermore, off-chip gelation is easier to result in loss of the encapsulated agents, since liquefied droplets have to be collected in separated vials before solidification, which will increase the diffusion of some encapsulated agents into the continuous oil phase [55].

Gelation of droplets to produce microgels can be broadly divided into chemical way and physical way [56]. Chemical gelation usually forms mechanically strong microgels, which is not easily enzymatically cleaved and metabolized. Furthermore harsh reaction conditions may be needed for synthesis of chemically cross-linkable pregel polymers [57]. On the other hand, physical gelation is generally mild and eliminates the use of toxic chemicals such as photoinitiators and glutaraldehyde [58].

\section{Photo Initiated Chemical Gelation}

Chemical gelation of the droplets can be induced by heat, UV, adding catalysts and use of crosslinking agents. Due to the use of optically clear polymer (e.g. PDMS) and glass, many microfluidic devices can be easily integrated with UV light source to allow the photo-initiated gelation of the droplets. The UV light activates photo-initiators in the droplets which causes monomers to link to each other and solidifies the droplets. UV-induced gelation of the droplets has been used to create microgels with interesting properties $[59,60]$. Choi et al. presented a study using microfluidic method for the production of monodisperse poly (ethylene glycol) (PEG) microspheres using continuous droplet formation and in situ photo-polymerization in microfluidic devices (Figure 3). By changing the interfacial tension between the two immiscible phases (hexadecane plus Span 80 and PEG-diacrylate plus photoinitiators) and their flow rate, the size of the PEG microgels $(45 \sim 95 \mu \mathrm{m})$ could be easily controlled. The in situ gelation of the droplets in their study is made possible
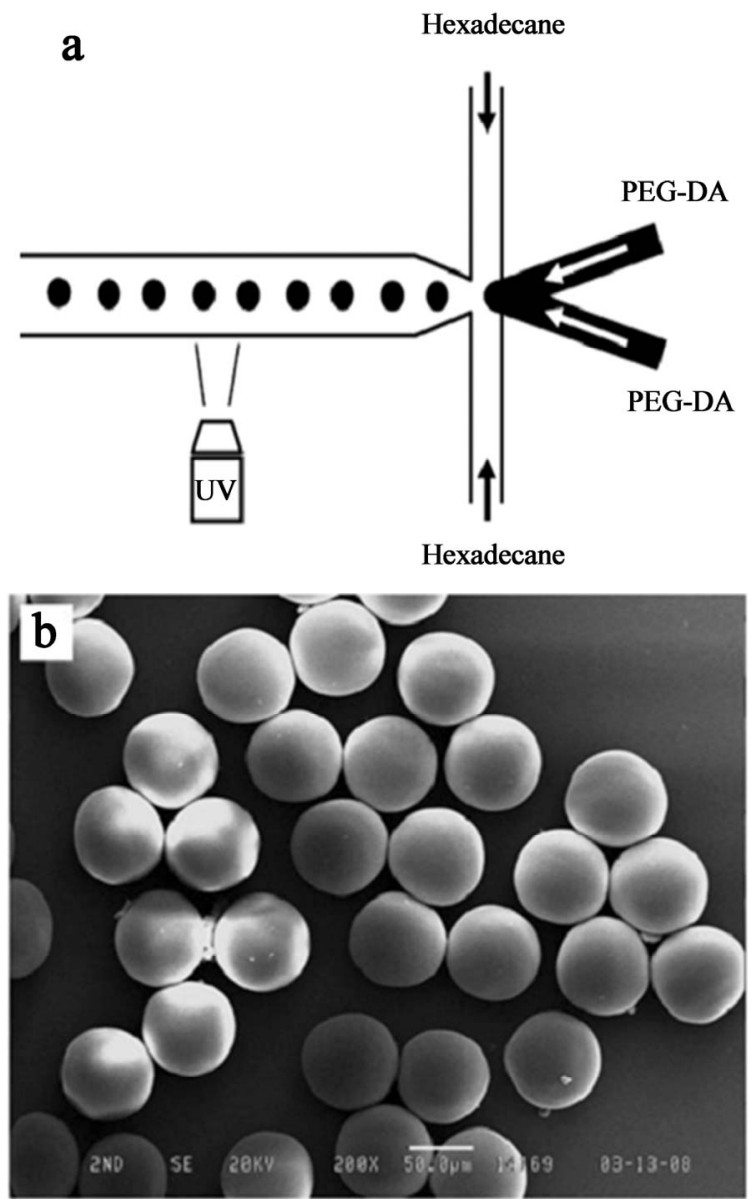

Figure 3. (a) Schematic of mirofluidic device for synthesizing monodisperse PEG microgels; (b) SEM image of PEG microgels produced from the microfluidic device (from ref. 61). 
by amplification of the UV light intensity through the high magnified objective lens. Under the conditions, PEG microgels could be rapidly solidified in very short exposure time ( $\sim 75 \mathrm{~ms})$ [61].

Besides the on chip UV gelation, off chip gelation was also carried out by De Geest et al. [62]. They used a flow focusing device with UV illumination to create dexHEMA microgels. The solution of dex-HEMA containing photo-initiator is sheared by the continuous mineral oil phase, and the droplets are collected along with the oil in a vial which is immediately irradiated with UV to solidify the droplets. They also tested the functionality of the microgels by incorporating FITC-BSA in the microgels to study the degradation of and release of protein from the microgels. One of the attractive advantages of dex-HEMA microgels is their biodegradability through hydrolysis of the dex-HEMA microgels leading to the formation of dextran chains and oligomethacrylates as degradation products [62].

Combining the UV curing and microfluidic channel geometry, non-spherical microgels, Janus microgels and multi-compartment microgels which exhibit interesting properties can also be synthesized [63-65]. Hwang et al. synthesized spherical and non-spherical PEG microgels in a T-junction microfluidic device containing an embedded UV light reflector. The reflector mode approach shows to enhance UV-based polymerization conditions for the synthesis of the microgels through an increased UV energy flux and a uniform distribution of the UV energy. In their design (Figure 4), monodisperse magnetic emulsion droplets are generated in a $\mathrm{T}$-junction and allowed to relax into spheres, disks, and plugs in confining microchannel geometries. The microgels geometry is locked-in via UV polymerization. The encapsulated magnetic nanoparticles give the microgels superparamagnetic behavior. Additionally, the non-spherical particles show anisotropic responses under an applied external magnetic field (Figure 4). These properties are quite desirable for the bottom-up modular tissue engineering, where multi cell types encapsulating in separated microgels can be assembled into functional tissues [63-65].

In order to make microgels having magnetic rotation ability, Chen et al. spatially patterned the wettability of the microfluidic channels to produce magnetic microgels with uniform anisotropic internal structure by a flow focusing drop maker using double emulsions as templates (Figure 5(a)) [66]. To achieve localized hydrophilicity, the hydrophobic PDMS microfluidic channels were filled with an aqueous monomer solution comprising acrylic with $\mathrm{NaIO}_{4}$ water, ethanol and acetone containing $10 \%$ benzophenone and then expose the channels under a focused spot of UV light to control the location where polyacrylic acid is grafted to the channel walls, making them locally hydrophilic (zone A in Figure 5(a)).

These microgels were produced by first producing oilin-water-in-oil $(\mathrm{O} / \mathrm{W} / \mathrm{O})$ double emulsion droplets, and then lock the anisotropic structure (magnetic cores) via photo-crosslinking the acrylamide monomers (Figure 5(b)). By adjusting the flow rate during formation, the size and internal structure of the particles, encapsulating one and two magnetic cores can be obtained (Figure 5(b)). One of the most appealing features of these microgels is their excellent rotational control by applying an external field, which can be used as micro-stirrer bars to mix fluids at small scales.

In addition to patterning hydrophilicity of the channels, a two-step process was also developed by Seiffert et al. to manufacture multi-compartment microgels $[67,68]$. To realize this, they make mono-dispersed hydrogel particles, and then wrap these particles in aqueous polymer shell using a PDMS device. The device consists of two crossjunctions in series, as shown in Figure 6(a). In the first junction, a semi-dilute, aqueous solution of crosslinkable pNIPAm chains as the shell phase was introduced. In the second junction, oil was added to form bi-layered pre-gel droplets and then crosslinking the pNIPAm shell. The

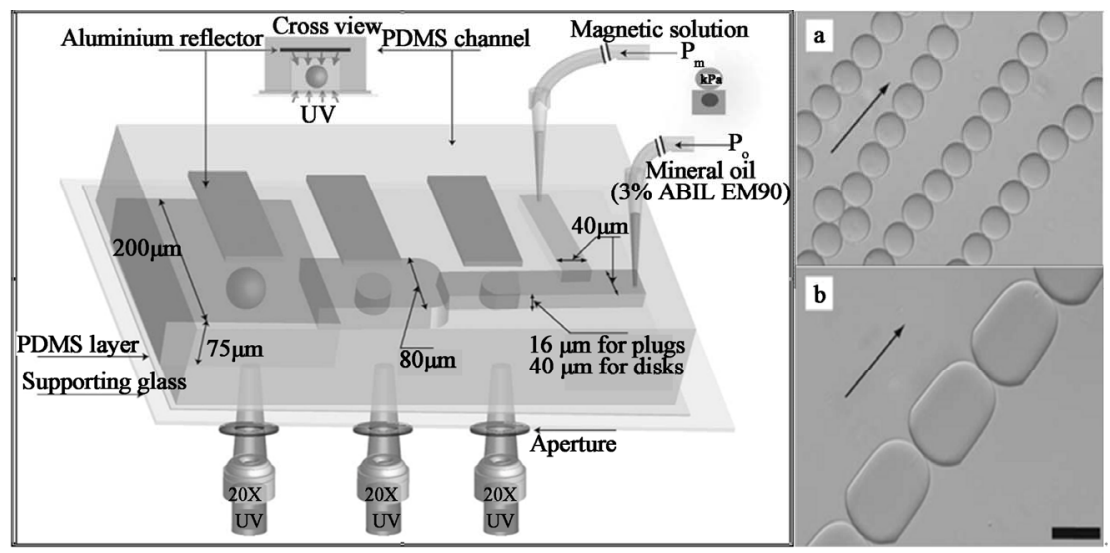

Figure 4. (left) Schematic of T-junction microfluidic channel with aluminum reflectors for spherical and non-spherical magnetic microgels synthesis; (right) (a) spheres \& (b) plugs in response to in-plane homogeneous magnetic field (from ref. 63). 


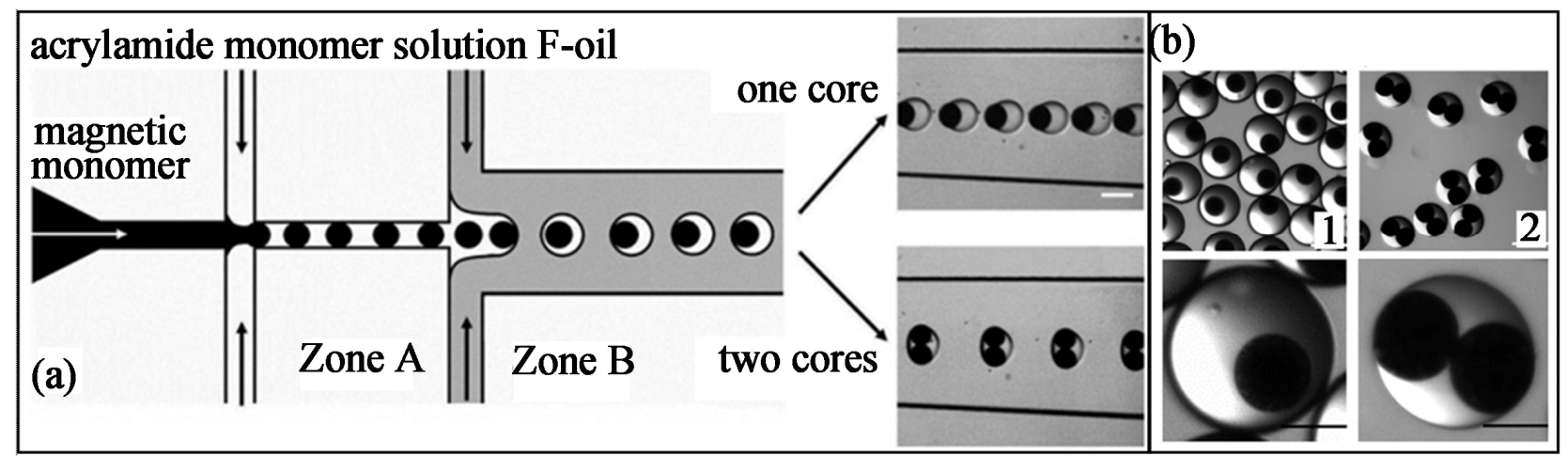

Figure 5. (a) Scheme of the PDMS device with patterned hydrophilicity for forming double emulsion droplets; (b) Microscope pictures showing the magnetic gel particles with uniform anisotropic feature. (1) microgels with single magnetic core; and (2) with two magnetic cores (from ref. 66 with permission (c) 2009 Wiley-VCH Verlag GmbH \& Co. KGaA, Weinheim).

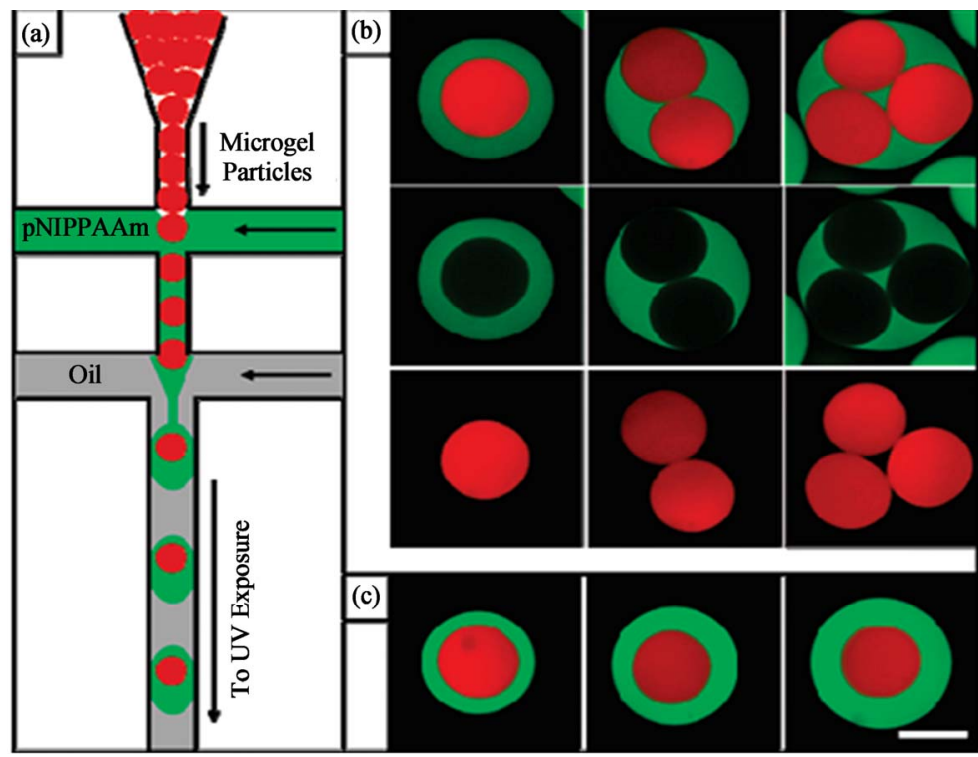

Figure 6. (a) Schematic of a microfluidic device forming aqueous pNIPAm droplets loaded with a well-defined number of pre-fabricated particles of a similar material, pNIPAm or polyacrylamide (b \& c) adjusting the flow rates of the inner particle phase, the middle polymer phase and the out oil phase controls the number of core particles in each shell (b) as well as (c) the shell thickness (from ref. 67 with permission (C) 2010 American Chemical Society).

obtained microgels consist of a hydrophilic polymer core that is nested in a hydrophilic polymer shell. Through adjusting the flow rate, microgel with single, double and triple cores can be obtained (Figures 6(b) and (c)). Stimuli-responsive size change of these microgels was demonstrated in their research. Non-thermo-responsive polyacrylamide (pAAm) microgel particles were loaded into pNIPAm shell to show the controlled release behaviors. The shell collapse due to the volume phase transition of pNIPAm, and the core remains unaffected in response to an increase of the temperature to $35^{\circ} \mathrm{C}[67,68]$.

Janus microgels synthesized from photo-initiation are also demonstrated in many studies. The term Janus microgels is used to describe micrgels particles having two distinct surface regions where different surface properties, different internal composition or different polarity are presented [69]. Shepherd et al. used microfluidic to assemble colloid-filled microgels of varying shape and composited. They first formed the drops by shearing a concentrated colloidal microsphere-acrylamide suspension in a continuous oil phase using a sheath-flow device (Figure 7). Both homogeneous and Janus microgels can be produced by confining the assembled drops in the micro-channels of varying geometry by the subsequent photo-polymerization. Their approach offers a facial route for assembling colloid-filled microgels with controlled shape and composition, which may lead to enhanced packing efficiency and novel application ranging from ceramics fabrication to pharmaceutical materials [70].

Recently, Seiffert et al. demonstrated a microfluidic technique to produce pNIPAm Janus microgels from pre- 
fabricated, cross-linkable pNIPAm precursor polymers. This approach separates the polymer synthesis from the particle gelation, thus allowing the droplet templating and functionalization of the matrix polymer to be performed and controlled in two independent steps (Figure 8(a)). The resultant microgels exhibits two distinguishable halves which contain most of the gelling precursors and the unmodified matrix polymer separate these materials (Figure 8(a)). Through using double emulsion droplets as templates, hollow Janus microgels can also be formed, indicating the great versatility of this method (Figure 8(b)) [71].

Besides colloid particles and dye molecules are encapsulated inside Janus microgels, functionalized viral nanotemplates are also loaded into Janus microgels to fabricate metabolically active microgels. Lewis et al. demonstrate rapid microfluidic fabrication of hybrid microgels composed of functionalized viral nanotemplates (tobacco mosaic virus) (TMV) directly embedded in the polymeric Janus microgels (Figure 9) [72]. Upon formation in a flow-focusing device, the droplets are photopolymerized with UV light to form Janus microgels. Catalytic activity, via the dichromate reduction reaction, is demonstrated with Janus microgels containing palladium-virus complex. Due to the compartmentation created by the Janus microgels, other functional nanoparticles like magnetic nanoparticles can also be embedded in other side of the Janus microgels, which enabled simple (a)

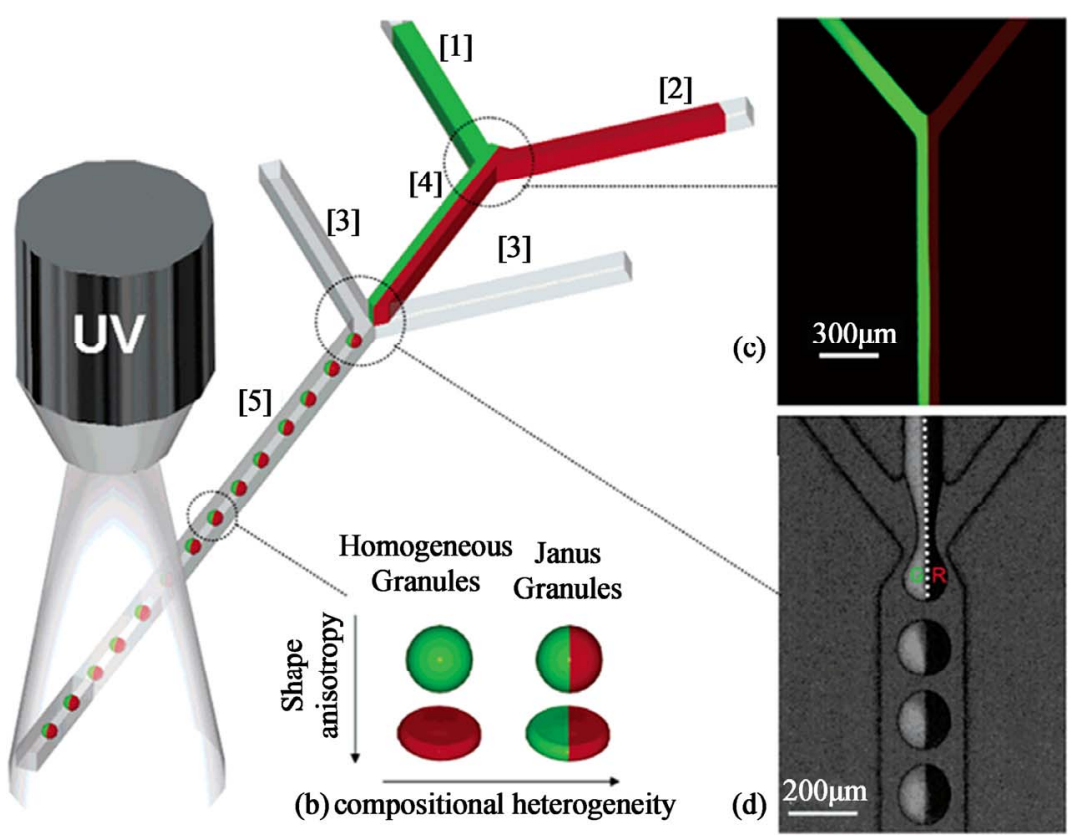

Figure 7. Schematic representation of sheath-flow microfluidic device used to produce monodisperse homogenous and Janus colloid-filled microgels (from ref. 69 with permission (c) 2006 American Chemical Society).
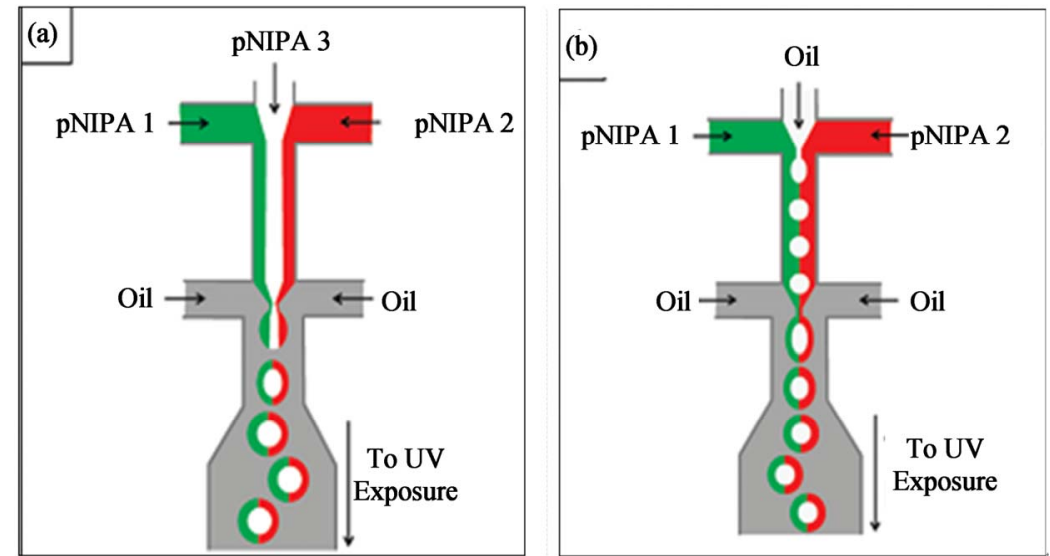

Figure 8. (a) Schematic of a microfluidic device forming aqueous droplets from three independent semi-dilute pNIPAm solution (b) Operating the device in a modified way yields oil-water-oil double emulsions with Janus-shaped middle phases (from ref. 71 with permission (C) 2010 American Chemical Society). 
separation of these microgels from bulk solution. Their results represent a facile route to directly harness the advantages of bioactive nano-template into a readily usable and stable 3D assembled format [72].

In addition to producing microgels with two phase system via photo-initiation, a stop-flow projection lithography method is alternatively developed by Doyle and co-workers to form microgels with single-phase microfluidic polymerization (Figure 10). Irradiation of the pre-gel solution through a mask with designed pattern caused site-specific crosslinking of PEG-DA. Various size and shape of microgels with monodispersity have been synthesized by this method. Some typical shapes of microgels are demonstrated in Figure 10. These mi-
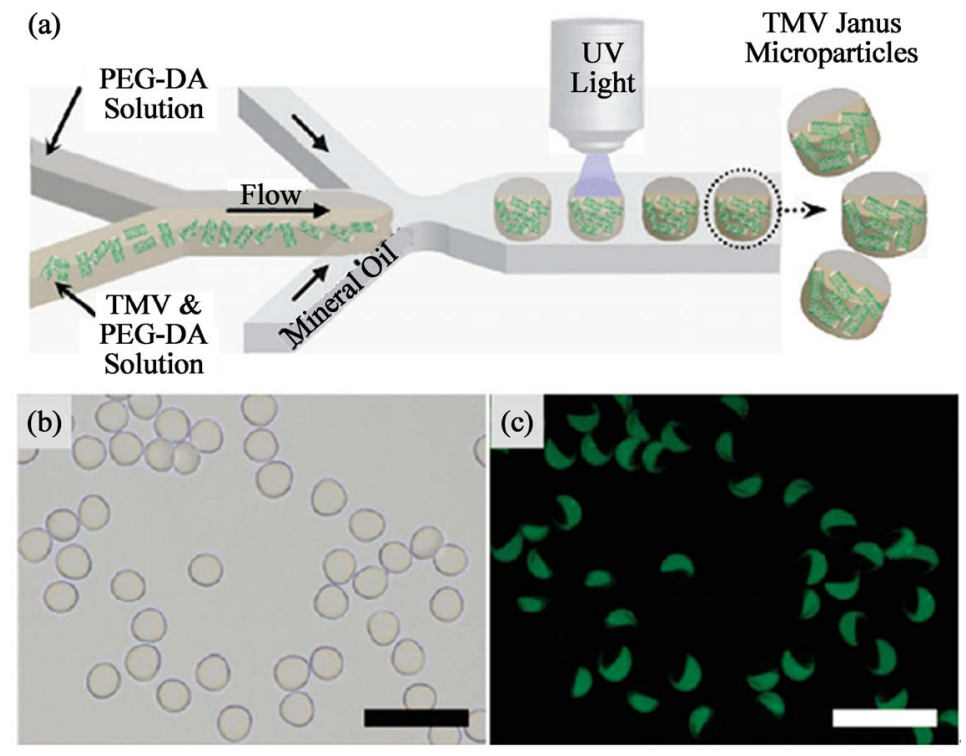

Figure 9. Microfluidic fabrication of Janus microgels containing fluorescently labeled TMV nanotemplates (a) Schematic diagram of Janus microgels synthesis in a microfluidic flow-focusing device; (b) bright field; and (c) fluorescence micrographs of Janus microgels with one side of the particles containing fluorescently labeled TMV (from ref. 72 with permission (c) 2010 American Chemical Society).
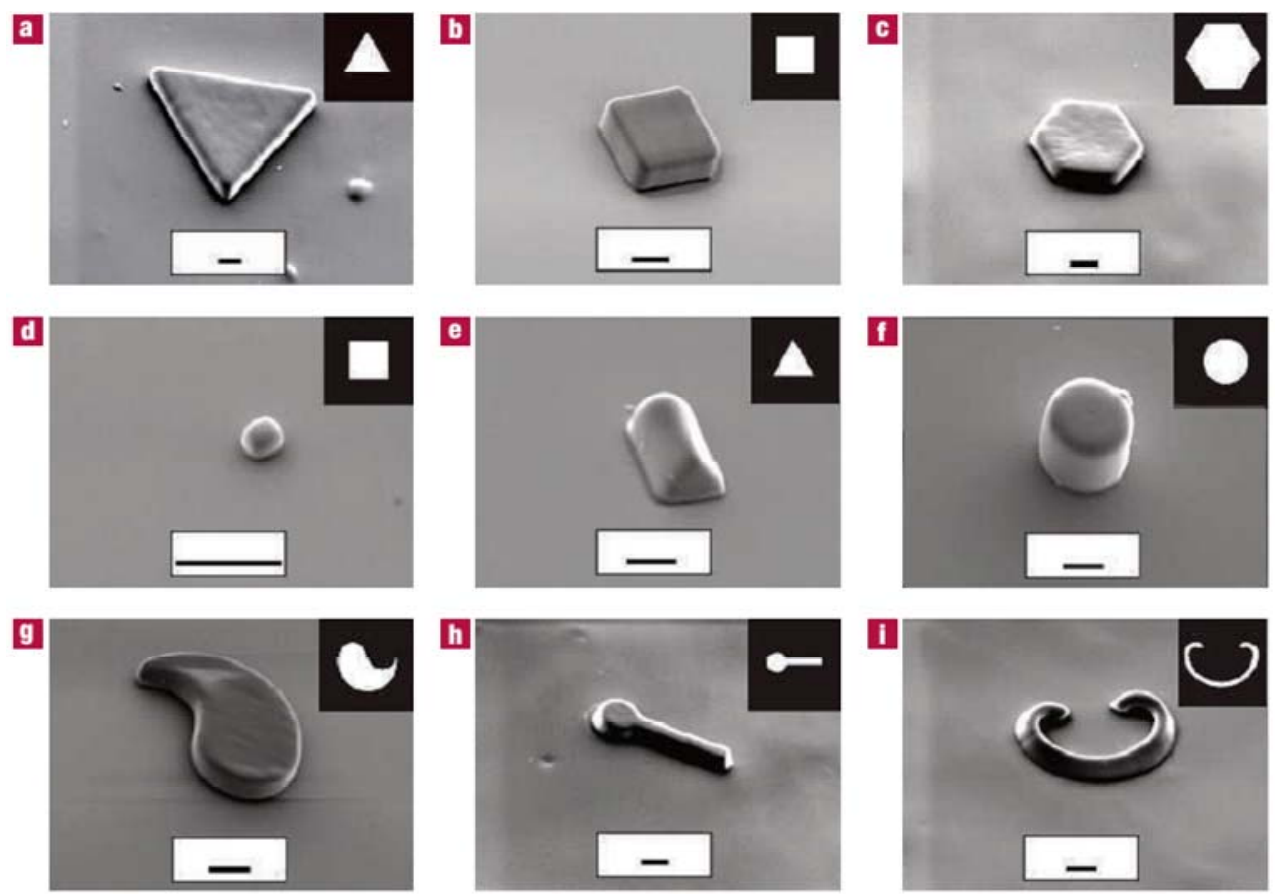

Figure 10. SEM images of particles (a - c) flat polygonal structures (d) colloidal buboid (e - $f$ ) high aspect ratio structure with different cross section ( $\mathrm{g}$ - i) curved particles (from ref. 73 with permission (c) Nature Publishing Group). 
crogels have great potentials of multi-functionality, providing unique features not found in homogeneous microgels [73].

\section{Non-Photo Initiated Chemical Gelation}

When using microgels for cell encapsulation or bioactive species loading, the UV-irradiation may be harmful to their activity, thereby limiting the encapsulation efficiency of this method $[74,75]$. Some improved methods such as limiting the UV-exposure time, addition of crosslinking accelerating agent and change to other wavelength instead of UV have been studied [74,75]. Some researchers also exploit radical-free method for the gelation of the droplets. Recently, Rossow et al. used nucleophilic Michael addition reaction to solidify the droplets, which shows beneficial features for the encapsulation of cells. In their design, they used a technique that combines droplet microfluidic templating with bioorthogonal thiol-end click reaction to fabricate monodisperse cell-laden microgels. The gelation of the droplets is achieved via the nucleophilic Michael addition of dithiolated PEG macro-cross-linkers to acrylated hyperbranched polyglycerol (hPG) building blocks and does not require any UV irradiation and initiator (Figure 11) [76]. Their synthesis of microgels from synthetic macromonomers provides more insight into the relation between the microgels properties and the viabilities of encapsulated cells. Meanwhile, the size, shape, and monodispersity of the microgels can be precisely controlled and a very mild, radical-free reaction for gelation of droplets is achieved [76].

Poly ( $N$-isopropylacrylamide) (pNIPAm) represents another import polymer material for the synthesis of microgels using chemical gelation method. One of the most attractive features of pNIPAm microgels is their thermo-responsive properties. A glass capillary sin- gleemulsion $(\mathrm{O} / \mathrm{W})$ device that consists of two sets of coflow geometries was used to produce pNIPAm microgels by Chu et al. (Figure 12(a)) [77-81]. An aqueous pre-gel mixture of NIPAm, bis-acrylamide (BIS), and ammonium persulfate is pumped through the cylindrical glass capillary and emulsified by a co-flowing oil phase of kerosene to form uniform droplets in the first co-flow geometry. The continuous phase containing the initiator, $N, N, N^{\prime}, N^{\prime}$-tetramethylethylenediamine (TEMED), is flowed between the outer coaxial region between the second square capillary and the right end of the tapered cylindrical capillary of the second co-flow geometry. The TEMED will diffuse into the droplets and thus induce the gelation of the polymers. The resultant microgels exhibit excellent thermal response (Figure 12(b)). By applying heating, their diameter is reduced to less than half the original diameter and the drugs are released out. This size change is reversible and reproducible over several

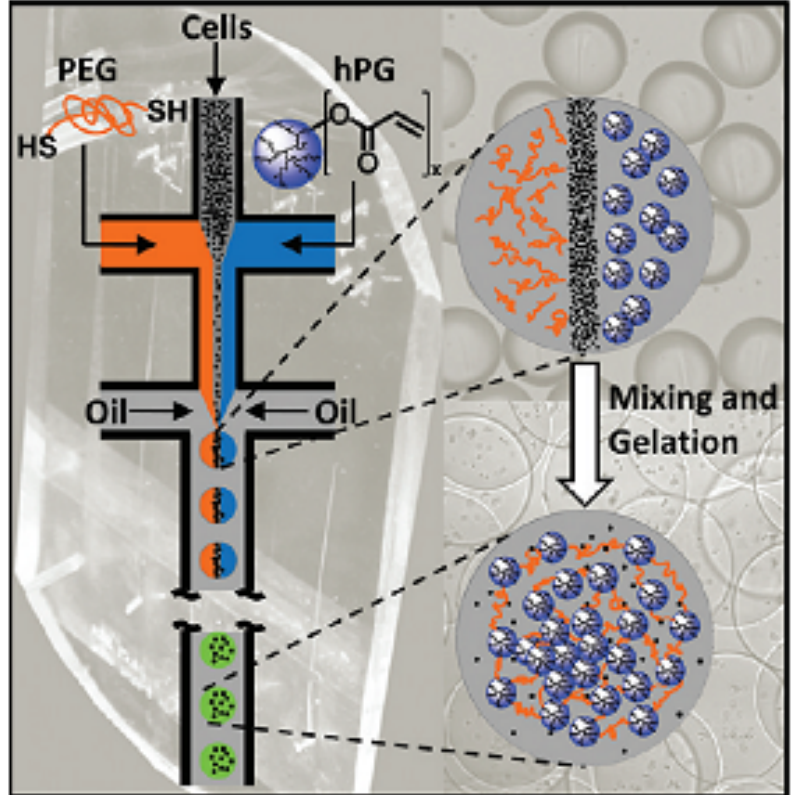

Figure 11. Microfluidic emulsification of aqueous solution containing dithiolated PEG macro-cross-linkers, acrylated hPG building blocks, and cells. Subsequent mixing of the three liquids inside the monodisperse droplets leads to droplet gelation by thiol-Michael addition of the macromonomers, thereby forming micrometer-sized cell laden microgels (from ref. 76 with permission (C) 2012 American Chemical Society).

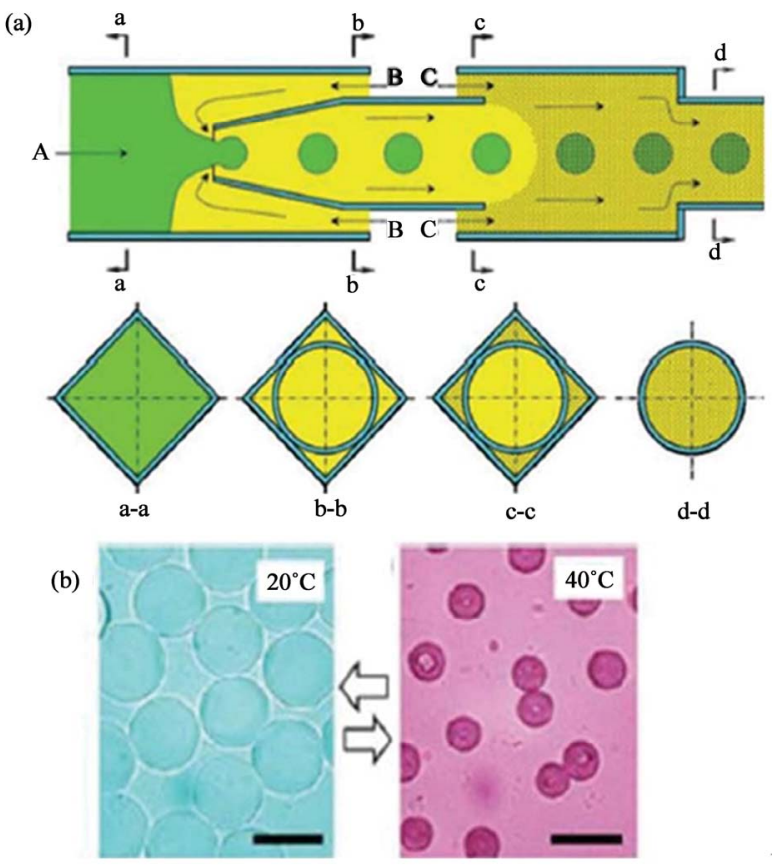

Figure 12. (a) Schematic illustration showing a capillary microfluidic device for fabrication of single compartment pNIPAm microgels. The cross-section at different points along the device is shown in the second row; (b) Size change of pNIPAm microgels in water triggered by changing the temperature (from ref. 77). 
cycles. Besides soluble drugs, fluorescent nanoparticles or drug-loaded nanoparticles can also be loaded into the interiors of the microgels, which can be used as multifunctional and multistage drug delivery systems [77-79].

Besides pNIPAm microgels with pNIPAm as the only component, pNIPAm microgels with an interpenetrating network structure is also synthesized by Raz and coworkers. In their study, microgels with a narrow size distribution are generated from ionically cross-linked alginate and chemically cross-linked pNIPAm (Figure 13) [80]. The Young's modulus and characteristic relaxation time of these microgels are studied using atomic force microscope (AFM). These microgels have the lower limits of the elasticity within the range of the elasticity of neutrophils. Consequently, these microgels can be a promising model system for studies of cell flow through constrained geometries [81,82].

Using bi-functional crosslinking agents like glutaraldehyde (GA), chemically crosslinked chitosan microgels are fabricated [83]. Jiang and co-workers create chitosan-bearing droplets by shearing off a chitosan solution at a microfluidic $\mathrm{T}$-junction with a stream of hexadecane containing GA (Figure 14). The chitosan contains amine groups that can readily react with aldehyde groups under mild conditions [84]. Chitosan microgels containing different components like magnetic nanoparticles and fluorescent nanoparticles can be readily fabricated by using GA as the crosslinking agent. Another important feature of GA is their ability to "glue" individual microgels. The on-chip connection of individual microgels into well-defined micro-chains is achieved using GA again to crosslink the amine groups in chitosan and confining the microgels into micro-channel as the spatial template (Figure 14). Chain flexibility can be tuned by adjusting the GA crosslinking time. These micro-assembled microgels chain may find application as microfluidic mixers, delivery vehicles, micro-scale sensor or miniature biomimetic robots. However, the cytotoxicity of GA need to be fully evaluated before applying microgels produced using this technique in biomedical fields. Less toxic crosslinking agents such as genipin may be an alternative choice [85].

\section{Physical Gelation}

Physical gelation is usually used for preparing microgels

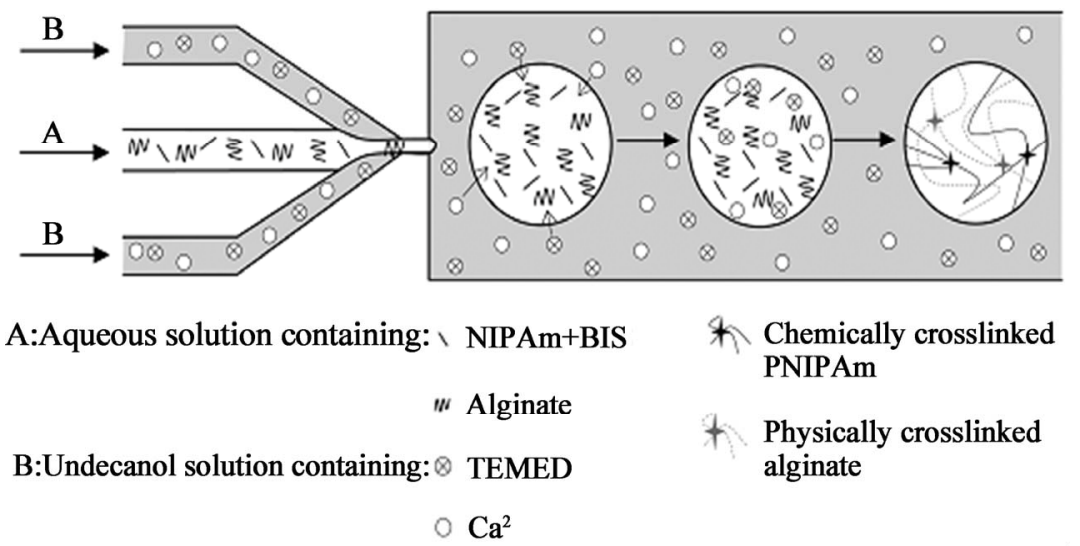

Figure 13. Schematic of microfluidic reactor for the synthesis of alginate-pNIPAm interpenetrate network microgels (from ref. 80 with permission (c) 2010 American Chemical Society).

(a) Partcle linking procedure

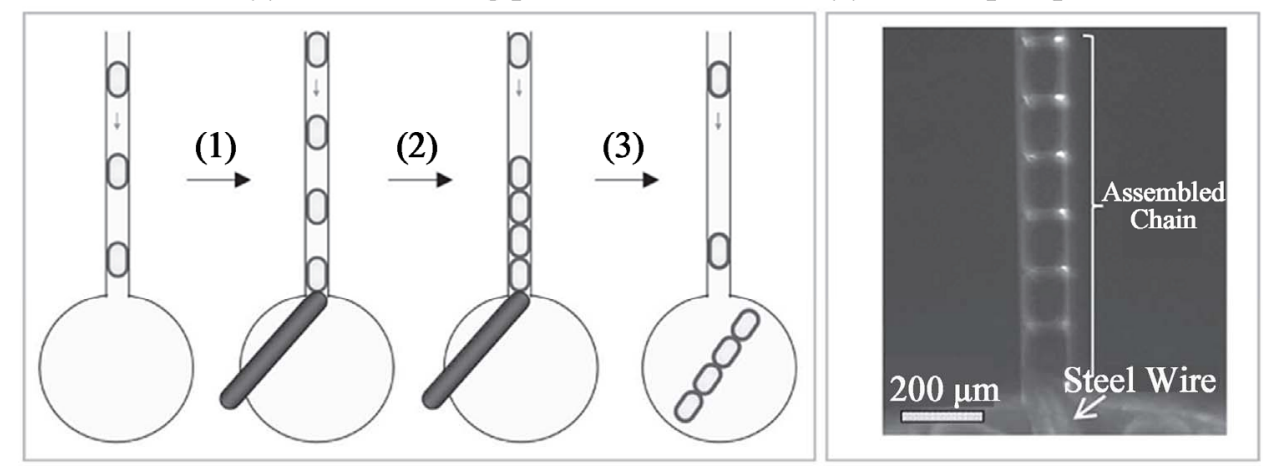

Figure 14. (a) Schematic depiction of linking individual chitosan microgels into chains; and (b) optical image showing the assembled chain inside the microchannel (from ref. 83). 
from natural polymers such as agarose, alginate, carrageenan, chitosan, gelatin, carboxymethylcellulose (CMC) and pectin. Gelation of these polymers can be induced by change of temperature and ionic crosslinking, where hydrophobic interaction, hydrogen bonding and ionic interaction are the major three forces for crosslinking the polymers $[86,87]$.

For temperature-induced gelation, the precursor polymer solution is emulsified at a temperature that is greater than the gelation temperature of the polymer. Cooling the droplets below the gelation temperature can obtain microgels particles. Gelatin and agarose are two typical thermally mediated gelation polymers [88,89]. Continuous on-chip emulsification and gelation of agarose microgels have been achieved by applying a temperature gradient to the microfluidic flow-focusing device. The syringe and upstream part of the devices for emulsification were maintained at a temperature above $37^{\circ} \mathrm{C}$, whereas the downstream channels were cooled to the lower temperature for agarose gelation. Similar design was also used to form k-carrageenan microgels [90].

Regarding the cell encapsulation application of microgels, collagen shows better feature than other gel material like PEG and agarose do. Because collagen is the most abundant scaffold protein in tissue and contains specific cell-binding sites that contribute to normal cell function [91]. There are few reports regarding the generation of collagen microgels in microfluidic devices. Until recently, an axisymmetric flow-focusing device (AFFD) is developed to generate collagen microgels. The mono-dispersed collagen microgels are generated at the orifice of a nozzle in the AFFD and collected in a test tube. The collected collagen micro-droplets are solidified at $37^{\circ} \mathrm{C}$ for $45 \mathrm{~min}$. There are some problems associated with this method. Due to the shearing forces generated from centrifugal extraction, the size and shapes of the final collagen microgels are non-uniform due to the coalescence of the micro-droplets [92].

In order to achieve on-chip temperate induced gelation of micro-droplets, Hong et al. proposed using an integrated microfluidic materials processing chip which integrates micro-droplets generation and gelation of the droplets on a single chip [93]. They use collagen as a demonstrative example. In their design, collagen droplets are generated at a microfluidic $\mathrm{T}$-junction between aqueous and mineral oil flows. The flow is heated immediately to $37^{\circ} \mathrm{C}$ to initiate collagen fiber assembly within a gelation channel. The microgels are extracted from the mineral oil into cell culture media within an extraction chamber. The collagen droplets solidify immediately after droplets generation significantly reduced coalescence. One advantage of this method is they can achieve higher microgels recovery and cell viability than the conventional off-chip centrifugation extraction approach.

Regarding the ionic gelling polymers, sodium alginate is the most widely studied candidate for microfluidic production of microgels. Coalescence-induced gelation, internal gelation and external gelation are the three major methods for producing alginate microgels [94]. For the coalescence-induced gelation of alginate, the process begins with the microfluidic emulsification of two types of droplets, on containing the alginate solution and the other one containing the crosslinking agents $\left(\mathrm{CaCl}_{2}\right)$ [94]. Coalescence of these two droplets in the downstream channel yields the solidified alginate microgels. This method is able to generate microgels with non-spherical shapes by coalescence occurring under geometric confinement of the pre-gel droplets in the microfluidic channels. For coalescence-induced gelation of alginate, complex design of microchannels and careful control of the flow rate to ensure coalescence happen within exact time is required. Furthermore, alginate microgels produced from such method have a wide and uncontrollable size distribution.

Figures 15(a)-(c) demonstrate the internal gelation of alginate microgels. In this method, microfluidic emulsification produces droplets containing alginate solution and $\mathrm{CaCO}_{3}$ nanoparticles in inactive state. In the continuous phase, acetic acid which is miscible with both the continuous phase and disperse phase is added. Due to the partition of acetic acid to the droplets, it dissolves the $\mathrm{CaCO}_{3}$ nanoparticles, the released $\mathrm{Ca}^{2+}$ are then trigger the polymer gelation [95]. One problem associated with the internal gelation method is the longer residence time of the microgels in the micro-channels is required to ensure the full crosslinking of the alginate micro-droplets, which will need longer gelation channel for this design, and thus limiting the microgels production throughput and increasing flow resistance. Another problem is the aggregation of $\mathrm{CaCO}_{3}$ nanoparticles in the feed tubing, causing loses of $\mathrm{CaCO}_{3}$ nanoparticles and blocking of the micro-channels [95].

For external gelation (Figures 15(d)-(f)) [96], droplets of alginate are generated with continuous oil phase containing the crosslinking agent which is also soluble in aqueous phases. As the droplets move through the downstream channels, the crosslinking agent (calcium acetate) diffuses into the droplets and thus initiates the gelation process. Microgels generated by external gelation method are stable upon their transfer into the cell culture medium. Comparing to the internal gelation method, external gelation provides better control of microgels morphology and microgels with capsular, gradient or uniform structure have been produced by external gelation method. 

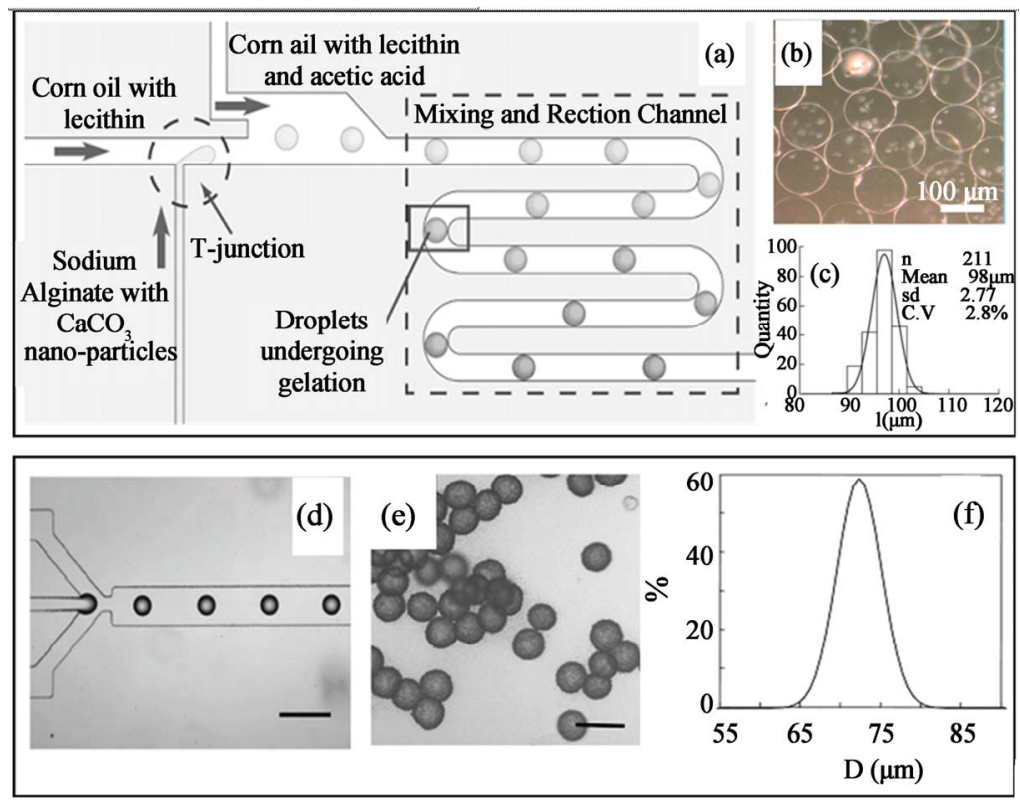

Figure 15. Internal gelation (a - c) and external gelation ( $d$ - $f$ ) of alginate droplets generated from microfluidic devices (a - c) (from ref. 95 with permission (c) 2007 Wiley-VCH Verlag GmbH \& Co. KGaA, Weinheim and (d - f) from ref. 96 with permission (C) 2006 American Chemical Society).

\section{Drug Delivery}

Unlike the conventional batch methods which result in production of poly-disperse microgels, microfluidic generation method can form microgels with reproducible size and fixed volume of aqueous medium, which provide a useful platform for therapeutic agents delivery. Control over particles size and minimization of the size distribution is up most important for the use of microgels in the route of administration and controlled release of the encapsulating compounds [97]. For example, Yang et al. encapsulated ampicillin into mono-dispersed chitosan microgels with microfluidic method. They found that the drug release rate could be controlled by the droplet size and due to the control over the small size distribution, these microgels offer potential for controlled-release drug delivery [98]. Jeong et al. reported a continuous fabriccation technique for manufacturing polymeric microgels containing biocatalysts. The active microgels initiate oxidase-catalyzed reactions have also been demonstrated. The fast generation and photo-polymerization process reduced the risk of chemical or optical damage to the entrapped enzyme and biological substrates, which is quite difficult to realize using the tradition bulk phase emulsion techniques. Multi-functional polycaprolactone (PCL) microparticles containing anti-cancer drug tamoxifen, $\mathrm{Fe}_{3} \mathrm{O}_{4}$ nanoparticles and $\mathrm{CdTe}$ quantum dots are also fabricated utilizing microfluidic emulsification, which combined magnetic targeting, fluorescence imaging and drug controlled release properties into one microparticle system (Figure 16) [99]. In addition to the great potential of multi-functionality of these microparticles, microfluidic production of these microparticles ensure the entire amount of tamoxifen could be entrapped in the PCL droplets without partitioning it into the immiscible phase, because $\mathrm{O} / \mathrm{W}$ emulsions are formed by shearing one liquid into a second immiscible one. The results of the tamoxifen release pattern (Figure 16) indicate that the release rate of the tamoxifen achieved the objective of the sustained release dosing protocol for clinical use.

Biocompatible and responsive microgels would be desirable for drugs delivery, since the drug release can be controlled in specific place in on-demand manner which can maximize the drug efficiency and diminish the side-effects. Various hydrophilic or hydrophobic drugs or even drug-loading nanoparticles sub-units have been loaded into the pre-gel droplets to form functional microgels. Responsive multi-compartment microgels have been prepared using higher order emulsions fabricated by using micro-capillary devices. Chu and co-workers used micro-capillary devices to prepare a triple emulsion, which can prepare monodisperse pNIPAm hydrogel microcapsules containing a multiphase core (Figures 17(a) and (b)). The resultant microgels consist of a shell of thermo-sensitive hydrogel, which encapsulate an oil core with different numbers of water droplets (Figures 17(c)-(g)). When the temperature increases, the pNIPAm gel rapidly shrinks by expelling water. The incompressible oil in the core, for which the hydrogel shell has lower permeability, exerts a count-stress on the inner wall of the shell. Consequently, the shell rupture and the encapsulated water droplets release out instantaneously. Dif- 

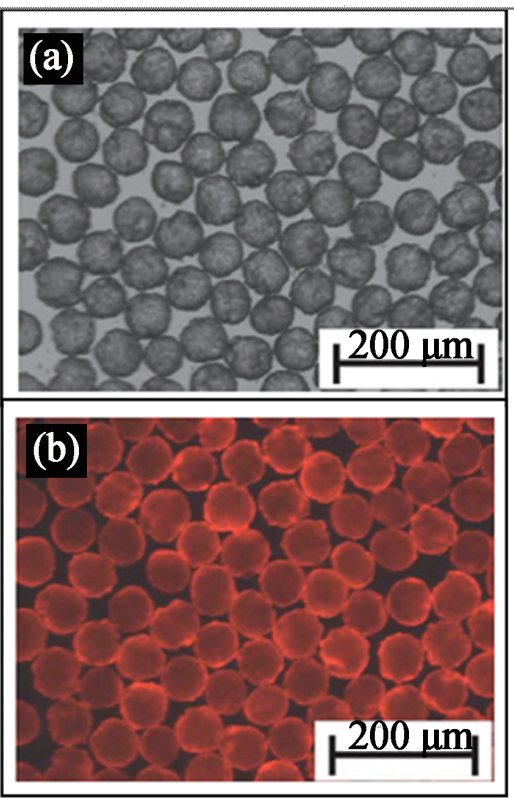

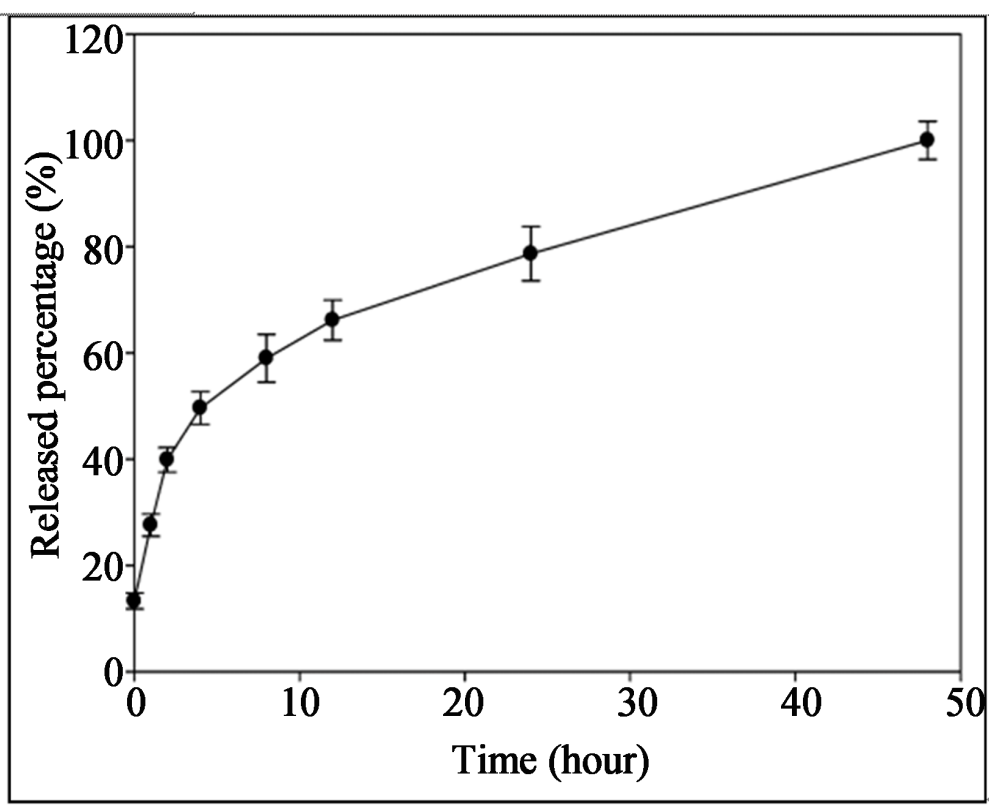

Figure 16. The photo images of the functional PCL microcapsules composed of tamoxifen, CdTe $\mathrm{QDs}_{\text {and }} \mathrm{Fe}_{3} \mathrm{O}_{4} \mathrm{NPs}$ synthesized from microfluidic device (a) bright field optical; (b) fluorescent microscope images; and (c) in vitro release profile of tamoxifen from the PCL microcapsules (from ref. 99).

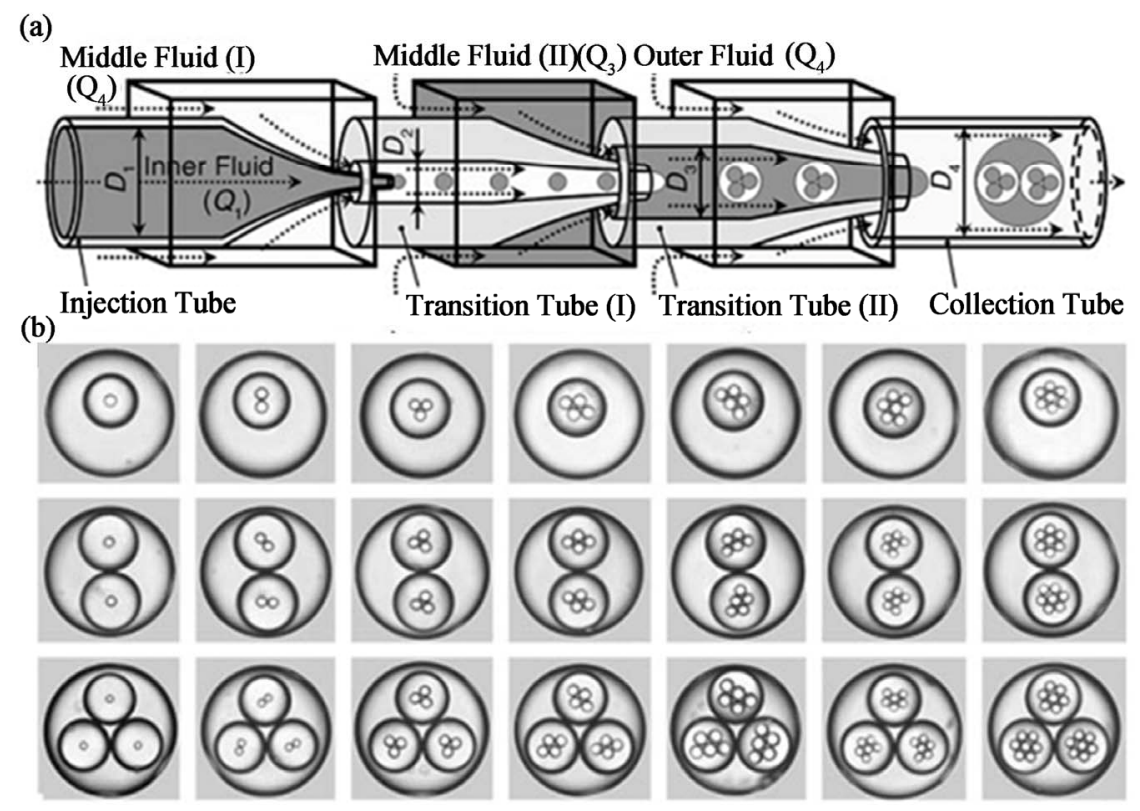

Figure 17. (a) Schematic diagram of the extended capillary microfluidic device for generating triple emulsion. (b) Optical micrographs of triple emulsion that contain a controlled number of inner and middle droplets. (c - g) optical micrograph of a microcapsule with a shell comprised of pNIPAm) containing multiple aqueous droplets in response to increased temperature (from ref. 100 with permission (c) 2007 Wiley-VCH Verlag GmbH \& Co. KGaA, Weinheim ).

ferent compounds such as hydrophobic drugs, hydrophilic drugs, functional nanoparticles like magnetic nanoparticles, Au, silver and fluorescent nanoparticles can be simultaneously loaded into the sub-units, which can build a multi-functional platform. Furthermore, such multicore microgels may also have important application as micro-reactor [100].

\section{Cell Encapsulation}

Encapsulation of cells in microgels has been demonstrated as an effective approach for cell immobilization, cell transplantation, artificial cell, cell therapy, bioassay and diabetes treatment. Control of cell numbers encapsulated in microgels particle, especially single cell encap- 
sulation in microgels is challenging yet quite needed in modern biomedical applications. For example, Courman and co-workers have demonstrated that single-cell microgels encapsulation can enhanced survival of human marrow stromal cells, which may markedly enhance therapeutic cell survival in targeted tissues [101].

Encapsulation of exact one cell per droplet with a high yield is challenging because the encapsulation is a random process and will follow Poisson statistics. Droplet microfluid, however, can beat the Poisson limitation and encapsulation of exact single cell per droplet with a high yield has been achieved by using high aspect ratios of microfluidic channels [102]. High aspect-ration microfluidic channels restrict cells to certain streamlines inside the channel and generate two trains of cells with a precisely staggered longitudinal spacing. This self-ordering phenomenon is utilized to generate drops with exact one cell. The self-organizing phenomenon will aid cell-indrop application where control of cell-occupancy is critical. Precise quantitative of secretion of uptake of trace amounts of bio-molecules by single cells can now be carried out more conveniently using this invention.

Instead of loading single cell type in respective microgels and then assembling them into macroscopic tissue, co-encapsulation of two or more cell types in individual microgels is a quite valuable technique for tissue engineering studies [103]. Tumarkin et al. presented a microfluidic platform for the high-throughput generation of microgels for cell co-culture [103]. Encapsulation of different cell types in the microgels is achieved by introducing in a microfluidic device two streams of distinct cell suspensions, emulsifying the mixed suspension, and gelling the pre-gel (agarose) droplets (Figure 18). Taking advantage of high degree of control endowed with microfluidic technique, different cell types at different ratio can be finely controlled within a single microgel, providing high throughput screening of cell co-culture conditions, leading to new strategies to manipulate cell fate. For example, co-encapsulation and co-culture of MBA2 and M07e cells at varying ratios demonstrated the ability of microfluidic approach to modulate paracrine signaling amongst cell population in a well-defined microenvironment. Co-encapsulation of MBA2 cells with UCB cells was also used to determined sub-populations of the hematopoietic cell types that are largely IL-3 dependent. Through understanding the controlled factor on the cells fate, modular tissue engineering involved different cell types assembly would have better control of cell morphology and bioactive behaviors, which will also provide a very useful in vitro model for the studying cell-cell interaction, drug screening, and tissue construction.

\section{Tissue Engineering}

Tissue engineering is an interdisciplinary research combing biology, medicine, material and engineering. Currently, there are mainly two approaches for the tissue engineering strategies, i.e. "top-down" approach and "bottom-up" approach with the former to be the more traditional strategy. In the "top-down" approach, isolated cells are seeded onto a biodegradable polymeric scaffold and populate the scaffold to create the appropriate extracellular matrix (ECM) with the aid of perfusion, growth factors and/or mechanical stimulation. One of the problems for this approach is the difficulty in recreating the intricate microstructural features of tissue. For the "bottom-up" approach (or named Modular Tissue Engineering), modular micro-tissue such as cell-laden microgels are assembled into engineered tissues with specific microarchitectural features. There is a strong biological basis for the bottom-up approach as many tissues

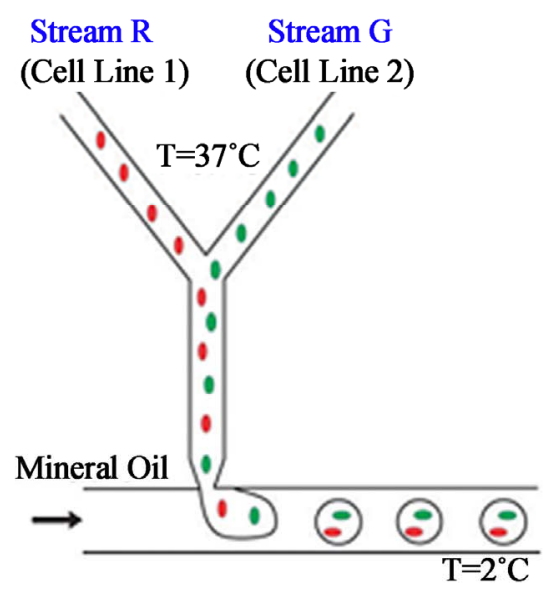

(a)

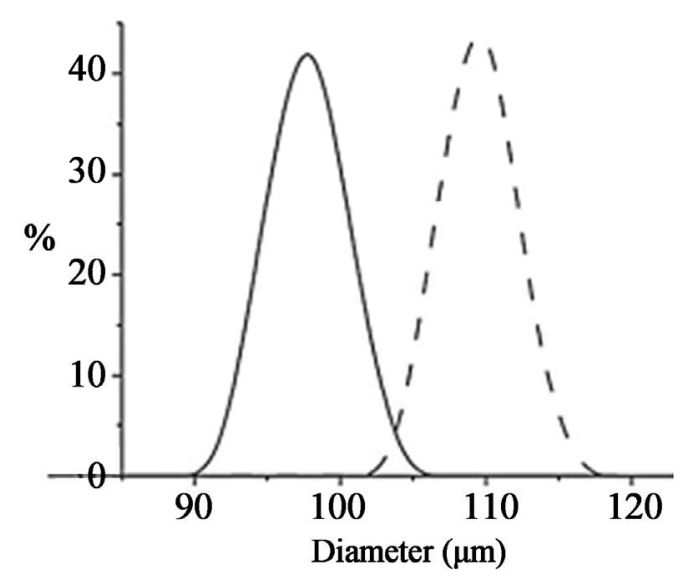

(b)

Figure 18. Microfluidic encapsulation of cells in agarose microgels (a) schematic of microfluidic cell co-encapsulation system (b) the distribution of sizes of the cell-laden droplets of $2 \mathrm{wt} \%$ agarose solution (dotted curve) and of the corresponding agarose microgels (solid curve) (from ref. 103). 
are comprised of repeating functional units and this approach can provide more guidance on the cellular level to direct tissue morphogenesis $[104,105]$. Microgels generated from droplet microfluidic techniques can have important contributions to both approaches but more studies are directed to exploit application of microgels in modular tissue engineering which can be regarded as more advantageous than the traditional top-down approach.

Monodispersity in size and shape of microgels produced from droplets microfluidic is one of the most attractive features, which can be also very beneficial for the top-down tissue engineering approach by acting as porous scaffold [106]. Recently, Lin and co-workers prepared alginate microgels filled with gas and used them as scaffold for cartilage tissue engineering. In their design (Figure 19(a)), the alginate droplets prepared from flow-focusing single emulsion were filled with gas and collected off-chip for gelation. Due to the monodispersed size, the collected alginate droplets will be orderly packed in a highly dense manner. After gelation in $\mathrm{CaCl}_{2}$ medium and vacuum degassing process, the alginate scaffold with highly interconnecting porous structure and uniform pore size was obtained (Figures 19(b)(f)). They found that this scaffold is effective in chondrocyte culture. The cell viability test, cell toxicity, cell survival rate, extracellular matrix production, cell proliferation and gene expression all revealed good results for chondrocyte culture. In comparison to the porous scaffold prepared by replicating from colloidal crystal, this method shows some advantage such as elimination of toxic chemicals, no extra step to clean the template colloidal crystal and finely-controlled pore size and connectivity. Furthermore, integrity of the generated scaffold from the droplet microfluidic technique can be fully preserved, which is quite difficult to achieve for the template colloidal crystal method, since the peeling of the formed scaffold from the template colloidal crystals can cause fracture of some connected pores due to the adhesion of scaffolding materials to the colloidal crystals $[107,108]$.

In addition to the "top-down" tissue engineering approach, more and more studies are focused on using cell laden microgels produced from microfluidic technique as building blocks for the modular tissue engineering. Through surface modification of the cell laden microgels with DAN molecules, cell laden microgels can be directly constructed into highly functional tissue-engineered implants [109]. Bhatia and co-workers modified the surface of cell laden PEG microgel with complementary DNA and form DNA-templated assembly of PEG microtissues. As Figure 20(a) shown, the recognition capabilities of DNA are the key to achieve rapid templated assembly of multiple microtissue types (Figure 20(a)). In their research, different cell laden microgels were respectively produced via single emulsion microfluidic droplet. These microgels were then modified with DNA-1. DNA-2, which is complemented with DNA-1 is patterned onto the glass substrate. The patterned glass substrates were then incubated with the cell laden microgels and then form the patterned microtissues (Figure 20(a)). Higher order of microtissues can be formed by using microgels containing different cell types (Figure 20(b)) [109].

Collagen, which is a more supportive material for cell encapsulation and growth than PEG, is also used to make cell laden microgels using microfluidic method for modular tissue engineering. Recently, Matsunaga et al. prepared mono-dispersed collagen microgels covered or encapsulated with cells using microfluidic technique (Figure 21) [110]. These functional microgels are then
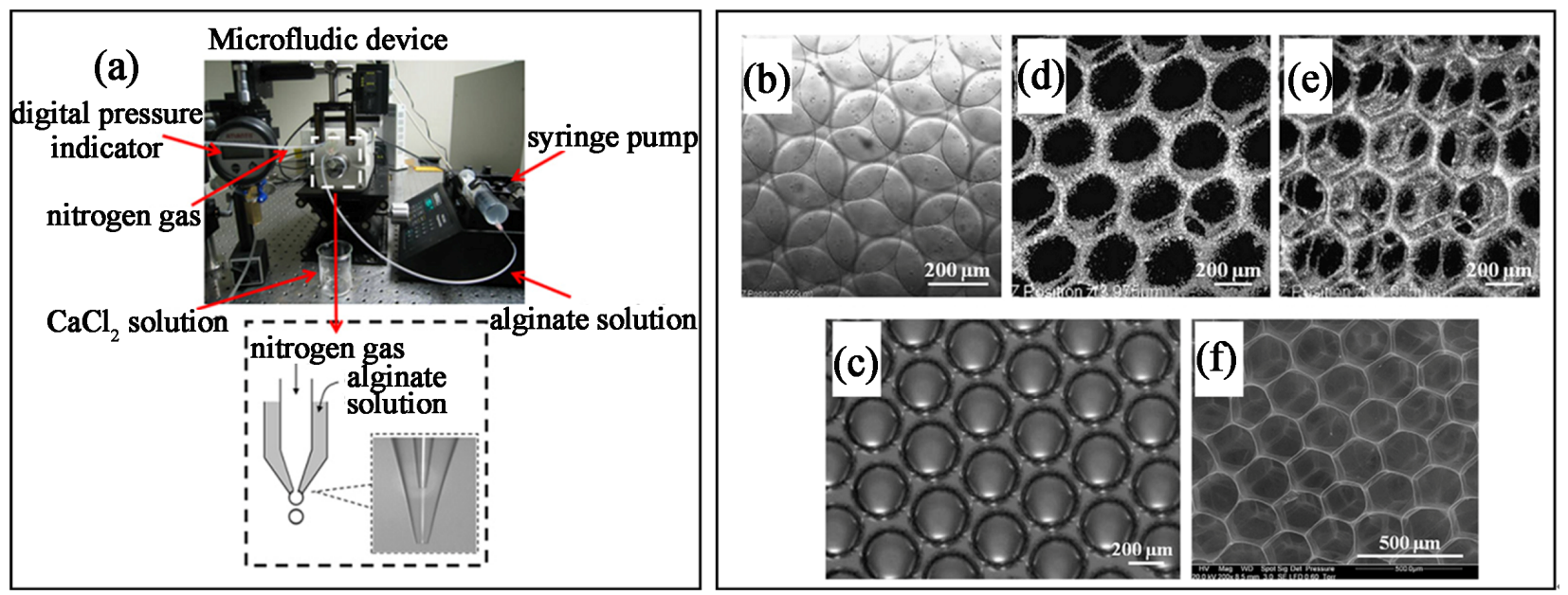

Figure 19. (a) Photograph showing the setup for preparing the air-filled alginate droplets; (b) monodisperse alginate bubbles generated from the microfluidic device; (c) the droplets formed a honeycomb structure after gelation; (d) - (f) confocal microscope showing a 3D ordered scaffold after vacuum degassing process (from ref. 106 with permission () 2011 Elsevier). 


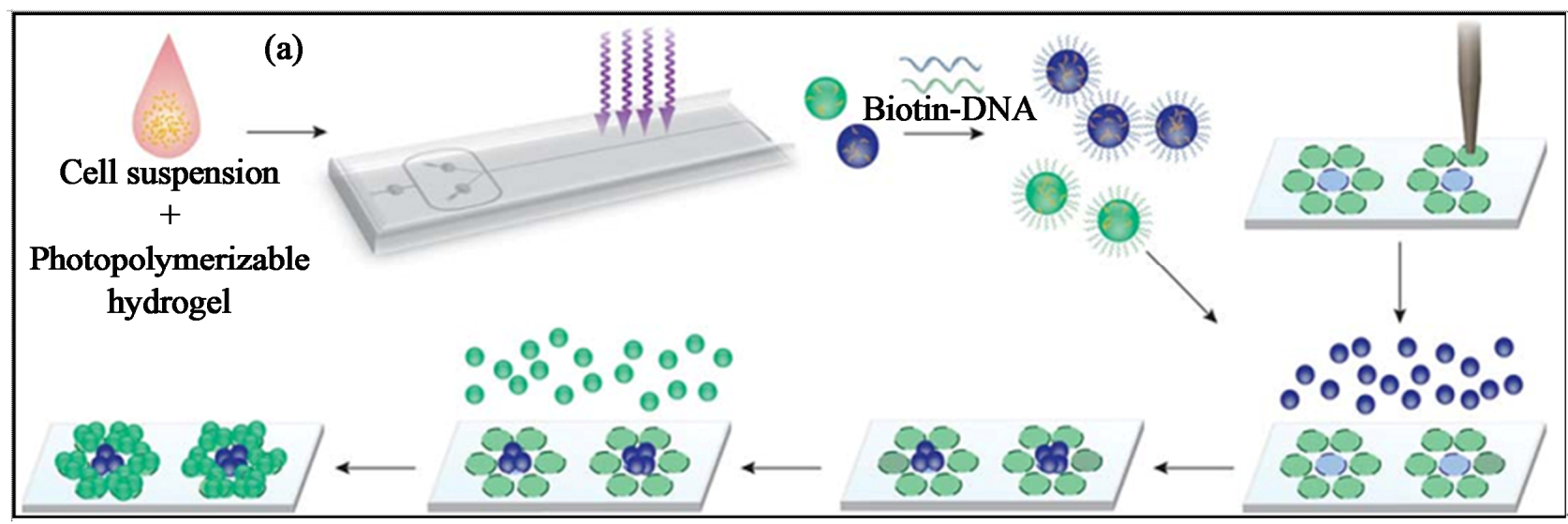

(a)

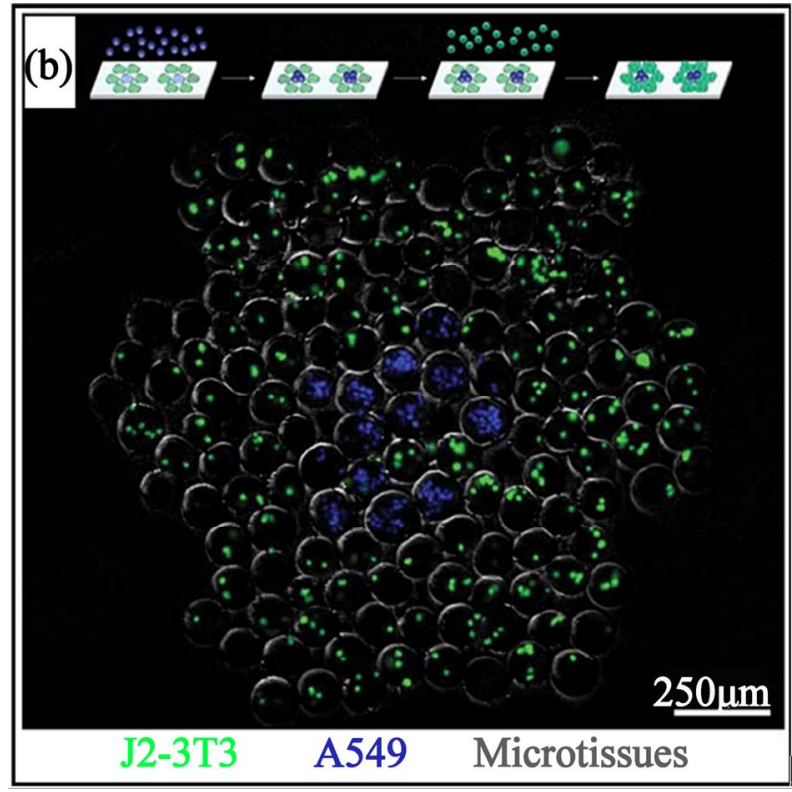

(b)

Figure 20. (a) scheme showing the production of microgels encapsulating different cells and their self-assembly into microtissue via DNA recognition (b) representative microscope pictures showing the microtissues encapsulating either J2-3T3 or A 543 cells assembling into specific shape (from ref. 109).

into a designed silicon chamber to form the macroscopic 3D tissue structure. In the mold, microgels having different cell types can adhere to each other via the cells coated on the collagen microgels. The cells can also migrate and grow into the collagen microgels, which lead to construction and decomposition of the collagen to ultimately form the macroscopic tissues. The benefit of using collagen instead of PEG is the biodegradability of collagen, which will help the formation of functional macroscopic tissues and is a key feature for the tissue engineering [111]. One of the important features for this method is the easy production of large numbers of mono-dispersed cell laden microgels of small diameter using the microfluidic technique. Furthermore, reconstructed 3D tissue with uniform cell density can be formed because the cell density of each microgels is tunable, which is impossible for the traditional top-down approach. In addition, using the cell laden microgels from microfluidic method, 3D tissue printing system can be easily integrated to achieve geometrical control of the cell laden microgels within the tissue.

\section{Conclusion and Outlook}

Droplet-based microfluidic production of microgels has displayed great advantages over the other methods in term of control of size, shape, chemistry, compartmenting and polarity. Enabling by the high degree of control offered by droplet microfluidic approach and its versatility, some novel microgels such as single cell encapsulated microgel and magnetically rotational microgels, which are difficult to fabricate using traditional methods, 
(a)

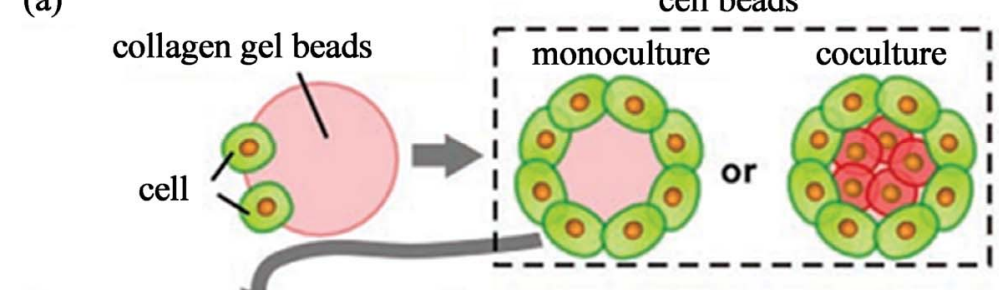

(b)

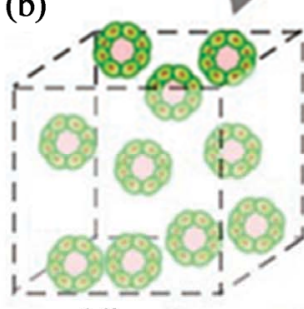

(c)

(d)

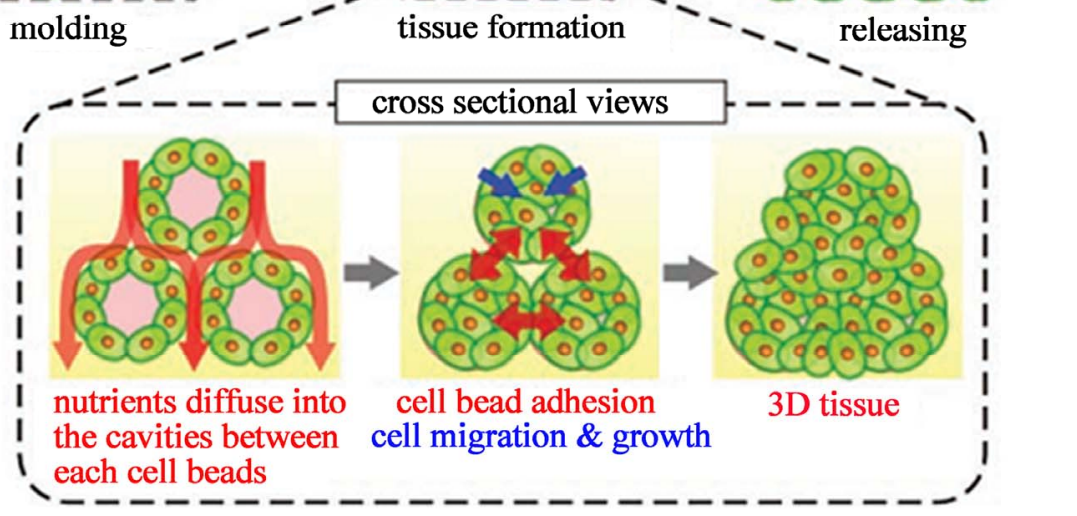

Figure 21. Concept of microgels-based tissue engineering: Monodisperse cell beads are molded into 3D tissue architecture (a) size-controlled cell beads are prepared by culturing cells over the surface of monodisperse collagen gel beads or collagen gel beads encapsulating another cell type; (b) cell bead are stacked into the designed silicon mold to form 3D tissues; (c) during tissue formation, the medium diffuse into the cavities between cell beads, supplying nutrients into the 3D constructs. The cells located on the surface of the beads form cell-cell connections. Cells migrated and grow within the collagen gel beads and finally form the 3D tissues; and (d) the 3D tissues are released from the mold (from ref. 110 with permission ${ }^{C} 2007$ Wiley-VCH Verlag GmbH \& Co. KGaA, Weinheim).

are now effectively achieved by droplet microfluidic techniques.

One of the important future developments for the production of microgels with droplet microfluidic will be the increased productivity. In a single microfluidic device, the production of microgels is limited by the intrinsically low productivity. Utilization of multiple parallel microfluidic reactors can show the ability to scale-up the microfluidic production of microgels. Further optimization of multiple parallel microfluidic devices would be the simultaneous production of microgels with different size and composition. Currently, parallel device consists of an array of $8 \times 12$ droplet makers is able to encapsulate 96 samples at the same time using air pressure to drive the fluid through the channel. Other driving forces like centrifugal force can also be a good candidate for parallel microfluidic device to scale-up the production of microgels.

The synthesis of smaller microgels having a size rang- ing from 3 to $25 \mu \mathrm{m}$ would be highly desirable. Previous experiments have showed the reduction of microgels size is achieved by narrowing the width and height of the channel nozzle. In order to form smaller microgels, nanofluidic devices may be desirable.

Another further effort for varying application of microgels would be extending the range of materials used in the microgels generation. Responsive hydrogel, interpenetrating polymer network (IPN) hydrogel and nano-composite hydrogel can be the three important candidates. Stimuli-responsive microgels enable the drug release in an as-demanded manner with precise concentration. Responsive microgels can be designed to be triggered by various stimuli such as $\mathrm{pH}$, ionic strength, temperature, magnetic force and light, which can greatly broaden the biomedical applications of microgels. One of the most features of IPN microgels will be their controllable elasticity, which can find important application as artificial red blood cell that need to pass through smaller 
diameter of capillary. Nano-composite hydrogel have found important application as tissue engineering scaffold and drug delivery carriers due to their robust mechanical properties. Production of nano-composite microgels with droplet microfluidic can further extend the biomedical application of microgels.

Regarding the application of microgels generated by microfluidic method, their biomedical application especially utilization as active cell laden units will be dominant application. Instead of loading limited dosage of drugs inside the microgels, encapsulating of cells secreting active compounds like peptide and enzymes enables the longer term safe therapeutic treatment in specific targeting location. Furthermore, cell based delivery system represents an intelligent delivery system which will secret targeting substance when receiving the stimuli from surrounding cells. Meanwhile, combination of producing microgels with microfluidic techniques, single cell encapsulation is an important direction. Due to the high sensitivity of single cell reaction in small volume of the microgels, co-encapsulation or injection (can integrate with paralleled pico-injector) of bioactive species such as enzymes, cytokines and drugs inside the microgels provides a new tool for exploring single cell genomics, proteomics and metabolomics. Enabling by the high degree of control from microfluidic methods, multicompartment or Janus microgels can be synthesized. These novel microgels can find important application as micro-reactor, stimuli triggered reaction vessel and in vitro model for drug screening. By isolating single cells or other active components in different compartments within a micro-size vessel, the reaction can become highly sensitive and finely controlled, which can enhance the analysis throughput. Another important direction of cell laden microgels would be using as building block in modular tissue engineering. Spherical cell laden microgels have been demonstrated their application in tissue engineering. Non-spherical or designable shape cell laden microgels which can self-assemble into complex macroscopic tissue would be quite beneficial for the modular tissue engineering.

\section{Acknowledgements}

We thank the financial supports from NUS startup grant (R-397-000-137-133) and SMART innovation grant (R397-000-146-592).

\section{REFERENCES}

[1] J. W. Kim, A. S. Utada, A. F. Nieves, Z. B. Hu and D. A. Weitz, "Fabrication of Monodisperse Gel Shells and Functional Microgels in Microfluidic Devices," Angewandte Chemie International Edition, Vol. 46, No. 11, 2007, pp. 1819-1822. doi:10.1002/anie.200604206

[2] Y. Qiu and K. Park, "Environment-Sensitive Hydrogels for Drug Delivery," Advanced Drug Delivery Reviews, Vol. 53, No. 3, 2001, pp. 321-339. doi:10.1016/S0169-409X(01)00203-4

[3] R. Langer and N. A. Peppas, "Advances in Biomaterials, Drug Delivery and Bionanotechnology," AIChE Journal, Vol. 49, No. 12, 2003, pp. 2990-3006.

doi:10.1002/aic.690491202

[4] S. Seiffert and D. A. Weitz, "Microfluidic Fabrication of Smart Microgels from Macromolecular Precusors," Polymer, Vol. 51, No. 25, 2010, pp. 5883-5889.

doi:10.1016/j.polymer.2010.10.034

[5] S. Abraham, E. H. Jeong, T. Arakawa, S. Shoji, K. C. Kim, I. Kim and J. S. Go, "Microfluidic Assisted Synthesis of Well-Defined Spherical Polymeric Microcapsules and Their Utilization as Potential Encapsulants," Lab Chip, Vol. 6, No. 6, 2006, pp. 752-756. doi:10.1039/b518006f

[6] G. F. Christopher and S. L. Anna, "Microfluidic Methods for Generating Continuous Droplets Stream," Journal of Physics D: Applied PhysicsEmail alert RSS feed, Vol. 40, No. 19, 2007, pp. R319. doi:10.1088/0022-3727/40/19/R01

[7] J. D. Wan, "Microfluidic-Based Synthesis of Hydrogel Particles for Cell Microencapsulation and Cell-Based Drug Delivery," Polymer, Vol. 4, No. 4, 2012, pp. 1084 1108. doi.10.3390./polymer4021084

[8] J. D. Wan, A. Bick, M. Sullivan, H. A. Stone, "Controllable Microfluidic Production of Microbubbles in Water-In-Oil Emulsion and the Formation of Porous Microparticles," Advanced Materials, Vol. 20, No. 17, 2008, pp. 3314-3318. doi:10.1002/adma.200800628

[9] M. Seo, Z. H. Nie, S. Q. Xu, M. Mok, P. C. Lewis, R. Graham and E. Kumacheva, "Continuous Microfluidic Reactors for Polymer Particles," Langmuir, Vol. 21, No. 25, 2005, pp. 11614-11622. doi:10.1021/la050519e

[10] D. Dendukuri and P. S. Doyle, "The Synthesis and Assembly of Polymeric Microparticles Using Microfluidics," Advanced Materials, Vol. 21, No. 41, 2009, pp. 1-16. doi:10.1002/adma.200803386

[11] B. Kintses, L. D. Van Vliet, S. R. A. Devenish and F. Holfelder, "Microfluidic Droplets: New Integrated Workflows for Biological Experiments," Current Opinion in Chemical Biology, Vol. 14, No. 5, 2010, pp. 548-555. doi:10.1016/j.cbpa.2010.08.013

[12] A. B. Theberge, F. Courtois, Y. Schaerli, M. Fischlechner, C. Abell, F. Hollfelder and W. T. S. Huck, "Microdroplets in Microfluidics: an Evolving Platform for Discoveries in Chemistry and Biology," Angewandte Chemie International Edition, Vol. 49, No. 34, 2010, pp. 58465868. doi:10.1002/anie.200906653

[13] R. L. Srinivas, S. C. Chapin and P. S. Doyle, "AptamerFunctionalized Microgel Particles for Protein Detection," Analytical Chemistry, Vol. 83, No. 23, 2011, pp. 91389145. doi:10.1021/ac202335u

[14] C. Berkland, K. Kim, D. W. Pack, "Precision Polymer Microparticles for Controlled-Release Drug Delivery," ACS Symposium Series, Vol. 879, Chapter 14, 2004, pp. 197-213.

[15] B. Rotman, "Measurement of Activity of Single Mole- 
cules of $\beta$-D-Galactosidase," Proceedings of the National Academy Science of USA, Vol. 47, No. 12, 1961, pp. 1981-1991. doi:10.1073/pnas.47.12.1981

[16] J. K. Oh, R. Drumright, D. J. Siewart and K. Matyjaszewski, "The Development of Microgels/Nanogels for Drug Delivery Application," Progress in Polymer Science, Vol. 33, No. 4, 2008, pp. 448-477. doi:10.1016/j.progpolymsci.2008.01.002

[17] H. C. Shum, A. R. Abate, D. lee, A. R. Studart, B. G. Wang, C. H. Chen, J. L. Thiele, R. K. Shah, A. Krummel and D. A. Weitz, "Droplet Microfluidic for Fabrication of Non-Spherical Particles," Macromolecular Rapid Communications, Vol. 31, No. 2, 2010, pp. 108-118. doi:10.1002/marc200900590

[18] M. Marquis, D. Renard, and B. Cathala, "Microfluidic Generation and Selective Degradation of Biopoly-merBased Janus Microbeads," Biomacromolecules, Vol. 13, No. 4, 2012, pp. 1197-1203. doi:10.1021/bm300159u

[19] B. K. Lee, Y. H. Yun, J. S. Choi, Y. C. Choi, J. D. Kim and Y. W. Cho, "Fabrication of Drug-Loaded Polymer Microparticles with Arbitrary Geometries Using a Piezoelectric Inject Printing System," International Journal of Pharmaceutics, Vol. 427, No. 2, 2012, pp. 305-310. doi:10.1016/j.ijpharm.2012.02.011

[20] F. Ikkai, S. Iwamoto, E. Adachi and M. Nakajima, "New Method of Producing Mono-Sized Polymer Gel Particles Using Microchannel Emulsification and UV Irradiation," Colloid and Polymer Science, Vol. 283, No. 10, 2005, pp. 1149-1153. doi:10.1007/s00396-005-1271-z

[21] Z. Q. Chang, C. A. Serra, M. Bouquey, L. Prat and G. Hadziioannou, "Co-Axial Capillaries Microfluidic Device for Synthesizing Size- and Morphology-Controlled Polymer Core-Polymer Shell Particles," Lab Chip, Vol. 9, No. 20, 2009, pp. 3007-3011. doi:10.1039/b913703c

[22] M. Zourob, S. Mohr, A. G. Mayes, A. Macaskill, N. Perezmoral, P. R. Fielden and N. J. Goddard, "A Micro-Reactor for Preparing Uniform Molecularly Imprinted Polymer Beads," Lab Chip, Vol. 6, No. 2, 2006, pp. 296-301. doi:10.1039/b513195b

[23] E. Brouzes, "Droplet Microfluidics for Single-Cell Analysis," Methods in Molecular Biology, Vol. 853, No. 10, 2012, pp. 105-139. doi.10.1007/978-1-61779-567-1 10

[24] H. Song, D. L. Chen, R. F. Ismagilov, "Reactions in Droplets in Microfluidic Channels," Angewandte Chemie International Edition, Vol. 45, No. 44, 2006, pp. 7336-7365. doi:10.1002/anie.200601554

[25] K. S. Huang, T. H. Lai and Y. C. Lin, "Manipulating the Generation of Ca-Alginate Microspheres Using Microfluidic Channels as a Carrier of Gold Nanoparticles," Lab Chip, Vol. 6, No. 7, 2006, pp. 954-957. doi.10.1039/B606424H

[26] P. Garstecki, M. J. Fuerstman, H. A. Stoen and G. M. Whiteside, "Formation of Droplets and Bubbles in a Microfluidic T-Junction-Scaling and Mechanism of Break-Up," Lab Chip, Vol. 6, No. 3, 2006, pp. 437-446. doi:10.1039/b510841a

[27] C. N. Baroun, F. Gallaire and R. Dangla, "Dynamics of Microfluidic Droplets," Lab Chip, Vol. 10, No. 16, 2010, pp. 2032-2045. doi:10.1039/c001191f
[28] Z. H. Nie, M. S. Seo, S. Q. Xu, P. C. Lewis, M. Mok, E. Kumacheva, G. M. Whitesides, P. Garstecki and H. A. Stone, "Emulsification in a Microfluidic Flow-Focusing Device: Effect of the Viscosities of the Liquids," Microfluid Nanofluid, Vol. 5, No. 5, 2008, pp. 585-594. doi:10.1007/s10404-008-0271-y

[29] M. Seo, I. Gorelikov, R. Williams and N. Matsuura, "Influence of Electrolyte Composition on the Photovoltaic Performance and Stability of Dye-Sensitized Solar Cells with Multiwalled Carbon Nanotube Catalysts," Langmuir, Vol. 26, No. 12, 2010, pp. 13855-13860. doi:10.1021/la100406p

[30] L. S. Roach, H. Song, R. F. Ismagilov, "Controlling NonSpecific Protein Adsorption in a Plug-Based Microfluidic System by Controlling Interfacial Chemistry Using Fluorous-Phase Surfactants," Analytical Chemistry, Vol. 77, No. 3, 2005, pp. 785-796. doi:10.1021/ac049061w

[31] J. W. Choi, D. K. Kang, H. Park, A. J. deMello and S. I. Chang, "High-Throughput Analysis of Protein-Protein Interactions in Picoliter-Volume Droplets Using Fluorescence Polarization," Analytical Chemistry, Vol. 84, No. 8, 2012, pp. 3849-3854.doi:10.1021/ac300414g

[32] S. Y. Jung, S. T. Retterer and C. P. Collier, "Interfacial Tension Controlled Fusion of Individual Femolitre Droplets and Triggering of Confined Chemical Reactions on Demand," Lab Chip, Vol. 10, No. 24, 2010, pp. 33733376. doi.10.1039/C0LC00376J

[33] M. Meier, J. Kennedy-Darling, S. H. Choi, E. M. Norstrom, S. S. Sisodia and R. F. Ismagilov, "Plug-Based Microfluidics with Defined Surface Chemistry to Miniaturize and Control Aggregation of Amyloidogenic Peptides," Angewandte Chemie International Edition, Vol. 48, No. 8, 2009, pp. 1487-1489.

[34] J. Clausell-Tormos, D. Lieber, J. C. Baret, A. Ei-Harrak, O. J. Miller, L. Frenz, J. Blouwolff, K. J. Humphry, S. Koster, H. Duan. C. Holtze, D. A. Weitz, A. D. Griffiths and C. A. Merten, "Droplet-Based Microfluidic Platforms for the Encapsulation and Screening of Mammalian Cells and Multicellular Organisms," Chemical Biology, Vol. 15, No. 7, 2008, pp. 427-437.

[35] A. P. Aijian, D. Chatterjee and R. L. Garrell, "Fluorinated Liquid-Enabled Protein Handing and Surfactant-Aided Crystallization for Fully in Situ Digital Microfluidic MADI-MS Analysis," Lab Chip, Vol. 12, No. 14, 2012, pp. 2552-2559. doi:10.1039/c2lc21135a

[36] C. Holtze, A. C. Rowat, J. J. Agresti, J. B. Hutchison, F. E. Angile, C. H. J. Schmitz, S. Koster, H. Duan, K. J. Humphry, R. A. Scanga, J. S. Johnson, D. Pisignano and D. A. Weitz, "Biocompatible Surfactants for Water-InFluorocarbon Emulsions," Lab Chip, Vol. 8, No. 10, 2008, pp. 1632-1639. doi:10.1039/b806706f

[37] C. H. Chen, A. Sarkar, Y. A. Song, M. A. Miller, S. J. Kim, L. G. Griffith, D. A. Lauffenburger and J. Y. Han, "Enhancing Protease Activity Assay in Droplet-Based Microfluidics Using a Biomolecule Concentrator," Journal of American Chemical Society, Vol. 133, No. 27, 2011, pp. 10368-10371. doi:10.1021/ja2036628

[38] J. C. Baret, "Surfactants in Droplet-Based Microfluidics," Lab Chip, Vol. 12, No. 3, 2012, pp. 422-433. 
doi:10.1039/c1lc20582j

[39] D. Velasco, E. Tumarkin and E. Kumacheva, "Microfluidic Encapsulation of Cells in Polymer Microgels," Small, Vol. 8, No. 11, 2012, pp. 1633-1642. doi:10.1002/smll.201102464

[40] E. Tumarkin and E. Kumacheva, "Microfluidic Generation of Microgels from Synthetic and Natural Polymers," Chemical Society Reviews, Vol. 38, No. 8, 2009, pp. 21612168. doi:10.1039/b809915b

[41] E. Kumacheva and P. Garstecki, "Microfluidic Reactors for Polymer Particles," John Wiley \& Sons, New York, 2011.

[42] T. Thorsen, R. W. Roberts, F. H. Arnold and S. R. Quake, "Dynamic Pattern Formation in a Vesicle-Generating Microfluidic Device," Physical Review Letters, Vol. 86, No. 18, 2001, pp. 4163-4166. doi:10.1103/PhysRevLett.86.4163

[43] C. Priest, S. Herminghaus and R. Seemann, "Generation of Monodisperse Gel Emulsion in a Microfluidic Device," Applied Physical Letters, Vol. 88, No. 2, 2006, 3 p. doi:10.1063/1.2164393

[44] A. R. Abate, A. Poitzsch, Y. Hwang, J. Lee, J. Czerwinska and D. A. Weitz, "Impact of Inlet Channel Geometry on Microfluidic Drop Formation," Physical Review E, Vol. 80, No. 2, 2009, 5 p. doi:10.1103/PhysRevE.80.026310

[45] I. Kobayashi, S. Mukataka and M. Nakajima, "Production of Monodisperse Oil-in-Water Emulsion Using a Large Silicon Straight-Through Microchannel Plate," Industrial \& Engineering Chemisrty Research, Vol. 44, No. 15, 2005, pp. 5852-5856. doi:10.1021/ie050013r

[46] I. Kobayashi, K. Uemura and M. Nakajima, "Formulation of Monodisperse Emulsions Using Submicron-Channel Arrays," Colloids and Surfaces A: Physicochemical and Engineering Aspects, Vol. 296, No. 1-3, 2007, pp. 285289. doi:10.1016/i.colsurfa.2006.09.015

[47] I. Kobayashi, M. Nakajima, H. Nabetani, Y. J. Kikuchi, A. Shohno and K. Satoh, "Preparation of Micron-Scale Monodisperse Oil-in-Water Microspheres by Microchannel Emulsification," Journal of the American Oil Chemists' Society, Vol. 78, No. 8, 2001, pp. 797-802. doi:10.1007/s11746-001-0345-5

[48] M. T. Guo, A. Rotem, J. A. and D. A. Weitz, "Droplet Microfluidics for High-Throughput Biological Assays," Lab Chip, Vol. 12, No. 12, 2012, pp. 2146-2155. doi:10.1039/c2lc21147e

[49] S. Y. Teh, R. Lin, L. H. Hung and A. P. Lee, "Droplet Microfluidics," Lab Chip, Vol. 8, No. 2, 2008, pp. 198220. doi:10.1039/b715524g

[50] H. N. Yow and A. F. Routh, "Formation of Liquid CorePolymer Shell Microcapsules," Soft Matter, Vol. 2, No. 11, 2006, pp. 940-949. doi:10.1039/b606965g

[51] T. Kanai, D. Lee, H. C. Shum and D. A. Weitz, "Fabrication of Tunable Spherical Colloidal Crystals Immobilized in Soft Hydrogels," Small, Vol. 6, No. 7, 2010, pp. 807-810. doi:10.1002/smll.200902314

[52] S. Seiffert, J. Dubbert, W. Richtering, D. A. Weitz, "Reduced UV Light Scattering in PDMS Microfluidic De- vices," Lab Chip, Vol. 11, No. 5, 2011, pp. 966-968. doi:10.1039/c01c00594k

[53] P. Panda, S. Ali, E. Lo, B. G. Chung, T. A. Hatton, A. Khademhosseini and P. S. Doyle, "Stop-Flow Lithography to Generate Cell-Laden Microgel Particles," Lab Chip, Vol. 8, No. 7, 2008, pp. 1056-1061. doi:10.1039/b804234a

[54] Y. Deng, N. Zhang, L. Zhao, X. Yu, X. Ji, W. Liu, S. Guo, K. Liu and Z. A. Zhao, "Rapid Purification of Cell Encapsulated Hydrogel Beads from Oil Phase to Aqueous Phase in a Microfluidic Device," Lab Chip, Vol. 11, No. 23, 2011, pp. 4117-4121. doi:10.1039/c1lc20494g

[55] Y. Morimoto, R. Tanaka and S. J. Takeuchi, "Construction of 3D, Layered Skin, Microsized Tissues by Using Cell Beads for Cellular Function Analysis," Advanced Healthcare Materials, 2012. doi:10.1002/adhm.201200189

[56] S. Dror, "Designing Cell-Compatible Hydrogels for Biomedical Application," Science, Vol. 336, No. 6085, 2012, pp. 1124-1128.

[57] J. W. Nichol and A. Khademhosseini, "Modular Tissue Engineering: Engineering Biological Tissue from the Bottom up," Soft Matter, Vol. 5, No. 7, 2009, pp. 1312 1319. doi:10.1039/b814285h

[58] B. G. Chung, K. H. Lee, A. Khademhosseini and S. H. Lee, "Microfluidic Fabrication of Microengineered Hydrogels and Their Application in Tissue Engineering," Lab Chip, Vol. 12, No. 1, 2012, pp. 45-59. doi:10.1039/c1lc20859d

[59] Y. M. Ren, B. Yu, H. L. Cong, Y. R. Ma, Z. Z. Ma and X. Yuan, "Preparation of Monodisperse PEG Microspheres by a T-Junction Microfluidic Chip," Advanced Materials Research, Vol. 465, No. 12, 2012, pp. 178-181. doi:10.4028/www.scientific.net/AMR.465.178

[60] Z. H. Nie, W. Li, M. Seo, S. Q. Xu and E. Kumacheva, "Janus and Ternary Particles Generated by Microfluidic Synthesis: Design, Synthesis and Self-Assembly," Journal of American Chemical Society, Vol. 128, No. 29, 2006, pp. 9408-9412. doi:10.1021/ja060882n

[61] C. H. Choi, J. H. Jung, T. S. Hwang and C. S. Lee, "In Situ Microfluidic Synthesis of Monodisperse PEG Microspheres," Macromolecular Research, Vol. 17, No. 3, 2009, pp. 163-167. doi:10.1007/BF03218673

[62] G. De Geest, J. P. Urbanski, T. Thorsen, J. Demeester and S. C. De Smedt, "Synthesis of Monodisperse Biodegradable Microgels in Microfluidic Devices," Langmuir, Vol. 21, No. 23, 2005, pp. 10275-10279. doi:10.1021/la051527y

[63] D. K. Hwang, D. Dendukuri and P. S. Doyle, "Microfluidic-Based Synthesis of Non-Spherical Magnetic Hydrogel Microparticles," Lab Chip, Vol. 8, No. 10, 2008, pp. 16401647. doi: $10.1039 / \mathrm{b} 805176 \mathrm{c}$

[64] C. A. Serra and Z. Chang, "Microfluidic-Assisted Synthesis of Polymer Particles," Chemical Engineering \& Technology, Vol. 31, No. 8, 2008, pp. 1099-1115. doi:10.1002/ceat.200800219

[65] K. W. Bong, S. C. Chapin and P. S. Doyle, "Magnetic Barcoded Hydrogel Microparticles for Multiplexed Detec- 
tion," Langmuir, Vol. 26, No. 11, 2010, pp. 8008-8014. doi:10.1021/la904903g

[66] C. H. Chen, A. R. Abate, D. Lee, E. M. Terentjev and D. A. Weitz, "Microfluidic Assembly of Magnetic Hydrogel Particles with Uniformly Anisotropic Structure," Advanced Materials, Vol. 21, No. 31, 2009, pp. 3201-3204. doi:10.1002/adma.200900499

[67] S. Seiffert, J. Thiele, A. R. Abate and D. A. Weitz, "Smart Microgels Capsules from Macromolecular Precursors," Journal of American Chemical Society, Vol. 132, No. 18, 2010, pp. 6606-6609. doi:10.1021/ja102156h

[68] K. J. Lee, J. Yoon and J. Lahann, "Recent Advances with Anisotropic Particles," Current Opinion in Colloid \& Interface Science, Vol. 16, No. 3, 2011, pp. 195-202. doi:10.1016/j.cocis.2010.11.004

[69] R. F. Shepherd, J. C. Conrad, S. K. Rhodes, D. R. Link, M. Marquez, D. A. Weitz and J. A. Lewis, "Microfluidic Assembly of Homogeneous and Janus Colloid-Filled Hydrogel Granules," Langmuir, Vol. 22, No. 21, 2006, pp. 8618-8622. doi:10.1021/la060759+

[70] Q. Wang, D. Zhang, H. B. Xu, X. L. Yang, A. Q. Shen and Y. J. Yang, "Microfluidic One-Step Fabrication of Radiopaque Alginate Microgels with in Situ Synthesized Barium Sulfate Nanoparticles," Lab Chip, Vol. 12, No. 22, 2012, pp. 4781-4786. doi:10.1039/c21c40740j

[71] S. Seiffert, M. B. Romanowsky and D. A. Weitz, "Janus Microgels Produced from Functional Precursor Polymers," Langmuir, Vol. 26, No. 18, 2010, pp. 14842-14847. doi:10.1021/la101868w

[72] C. L. Lewis, Y. Lin, C. X. Yang, A. K. Manocchi, K. P. Yuet, P. S. Doyle and H. M. Yi, "Microfluidic Fabrication of Hydrogel Microparticles Containing Functionalized Viral Nanotemplates" Langmuir, Vol. 26, No. 16, 2010, pp. 13436-13441. doi:10.1021/la102446n

[73] D. Dendukuri, D. C. Pregibon, J. Collins, T. A. Hatton and P. S. Doyle, "Continuous-Flow Lithography for HighThroughput Microparticle Synthesis," Nature Materials, Vol. 5, No. 5, 2006, pp. 365-369. doi.10.1038/nmat1617

[74] G. J. M. Fechine, J. A. G. Barros and L. H. Catalani, "Poly(N-Vinyl-2-Pyrrolidone) Hydrogel Production by Ultraviolet Radiation: New Methodologies to Accelerate Crosslinking," Vol. 45, No. 14, Polymer, pp. 4705-4709. doi:10.1016/j.polymer.2004.05.006

[75] S. A. Lee, S. E. Chung, W. Park, S. H. Lee and S. H. Kwon, "Three-Dimensional Fabrication of Heterogeneous Microstructures Using Soft Membrane Deformation and Opofluidic Maskless Lithography," Lab Chip, Vol. 9, No. 12, 2009, pp. 1670-1675. doi:10.1039/b819999j

[76] T. Rossow, J. A. Heyman, A. J. Ehrlicher, A. Langhoff, D. A. Weitz, R. Haag and S. Seiffert, "Controlled Synthesis of Cell-Laden Microgels by Radical-Free Gelation in Droplet Microfluidics," Journal of American Chemical Society, Vol. 134, No. 10, 2012, pp. 4983-4989. doi:10.1021/ja300460p

[77] R. K. Shah, J. W. Kim, J. J. Agresti, D. A. Weitz and L. Y. Chu, "Fabrication of Monodisperse Thermosensitive Microgels and Gel Capsules in Microfluidic Devices," Soft Matter, Vol. 4, No. 12, 2008, pp. 2303-2309. doi: $10.1039 / \mathrm{b} 808653 \mathrm{~m}$
[78] L. Y. Chu, J. W. Kim, R. K. Shah and D. A. Weitz, "Monodisperse Thermoresponsive Microgels with Tunable Volume-Phase Transition Kinetics," Advanced Functional Materials, Vol. 17, No. 17, 2007, pp. 3499-3504. doi:10.1002/adfm.200700379

[79] W. J. Duncanson, T. Lin, A. R. Abate, S. Seiffert, R. K. Shah and D. A. Weitz, "Microfluidic Synthesis of Advanced Microparticles for Encapsulation and Controlled Release," Lab Chip, Vol. 12, No. 12, 2012, pp. 2135-2145. doi:10.1039/c2lc21164e

[80] N. Raz, J. K. Li, L. K. Fiddes, E. Tumarkin, G. C. Walker and E. Kumacheva, "Microgels with an Interpenetrating Network Structure as a Model System for Cell Studies," Macromolecules, Vol. 43, No. 17, 2010, pp. 7277-7281. doi:10.1021/ma101231z

[81] P. Roca-Cusachs, I. Almendros, R. Sunyer, N. Gavara, R. Farre and D. Navajas, "Rheology of Passive and Adhesion-Activated Neutrophils Probed by Atomic Force Microscopy," Biophysical Journal, Vol. 91, No. 9, 2006, pp. 3508- 3518. doi:10.1529/biophysj.106.088831

[82] E. P. Wojcikiewicz, X. H. Zhang, A. Chen and V. T. Moy, "Contributions of Molecular Binding Events and Cellular Compliance to the Modulation of Leukocyte Adhesion," Journal of Cell Science, Vol. 116, No. 15, 2003, pp. 2531-2539. doi: $10.1242 /$ jcs.00465

[83] K. Q. Jiang, C. Xue, C. Arya, C. R. Shao, E. O. George, D. L. DeVoe and S. R. Raghavan, "A New Approach to in Situ Micromanufacturing: Microfluidic Fabrication of Magnetic and Fluorescent Chains Using Chitosan Microparticles as Building Blocks," Small, Vol. 7, No. 17, 2011, pp. 2470-2476.

[84] R. C. Luo, B. Neu and S. S. Venkatraman, "Surface Functionalization of Nanoparticles to Control Cell Interactions and Drug Release," Small, Vol. 8, No. 16, 2012, pp. 25852594. doi:10.1002/smll.201200398

[85] R. A. A. Muzzarelli, "Genipin-Crosslinked Chitosan Hydrogels as Biomedical and Pharmaceutical Aids," Carbonhydrate Polymers, Vol. 77, No. 1, 2009, pp. 1-9. doi:10.1016/j.carbpol.2009.01.016

[86] M. Rubinstein and R. H. Colby, "Polymer Physics," Oxford University Press, Oxford, 2003.

[87] S. Ladet, L. David and A. Domard, "Multi-Membrane Hydrogels," Nature, Vol. 452, No. 6, 2008, pp. 76-80. doi:10.1038/nature06619

[88] S. Q. Xu, Z. H. Nie, M. Seo, P. Lewis, E. Kumacheva, H. A. Stone, P. Garstecki, D. B. Weibel, I. Gitlin and G. M. Whitesides, "Generation of Monodisperse Particles by Using Microfluidics: Control over Size, Shape and Composition," Angewandte Chemie International Edition, Vol. 44, No. 5, 2005, pp. 724-728. doi:10.1002/anie.200462226

[89] S. Iwamoto, K. Nakagawa, S. Sugiura and M. Nakajima, "Preparation of Gelatin Microbeads with a Narrow Size Distribution Using Microchannel Emulsification," AAPS PharmSciTech, Vol. 3, No. 3, 2002, pp. 72-76. doi:10.1007/BF02830623

[90] B. Walther, C. Cramer, A. Tiemeyer, L. Hamberg, P. Fischer, E. J. Windhab and A. M. Hermansson, "Drop Deformation Dynamics and Gel Kinetics in a Co-Flowing 
Water-in-Oil System," Journal of Colloid and Interface Science, Vol. 286, No. 1, 2005, pp. 378-386. doi:10.1016/j.jcis.2005.01.054

[91] G. A. Di Lullo, S. M Sweeney, J. Korkko, L. Ala-Kokko and J. D. San Antonio, "Mapping the Ligand-Binding Sites and Disease-Associated Mutations on the Most Abundant Protein in the Human, Type I Collagen," Journal of Biological Chemistry, Vol. 277, No. 8, 2002, pp. 4223-4230. doi:10.1074/jbc.M110709200

[92] Y. Morimoto, W. H. Tan and S. Takeuchi, Biomed. "ThreeDimensional Axisymmetric Flow-Focusing Device Using Stereolithography," Microdevices, Vol. 11, No. 2, 2009, pp. 369-377. doi:10.1007/s10544-008-9243-y

[93] S. M. Hong, H. J. Hsu, R. Kaunas and J. Kameoka, "Collagen Microsphere Production on a Chip," Lab Chip, Vol. 12, No. 18, 2012, pp. 3277-3280. doi:10.1039/c2lc40558j

[94] S. Sugiura, T. Oda, Y. Izumida, Y. Aoyagi, M. Satake, A. Ochiai, N. Ohkohchi and M. Nakajima, "Size Control of Calcium Alginate Beads Containing Living Cells Using Micro-Nozzle Array," Biomaterials, Vol. 26, No. 16, 2005, pp. 3327-3331. doi:10.1016/j.biomaterials.2004.08.029

[95] W. H. Tan, and S. Takeuchi, "Monodisperse Alginate Hydrogel Microbeads for Cell Encapsulation," Advanced Materials, Vol. 19, No. 18, 2007, pp. 2696-2701. doi:10.1002/adma.200700433

[96] H. Zhang, E. Tumarkin, R. Peerani, Z. Nie, M. A. Sullan, G. C. Walker and E. Kumacheva, "Microfluidic Production of Biopolymer Microcapsules with Controlled Morphology," Journal of American Chemical Society, Vol. 128 , No. 37, 2006, pp. 12205-12210. doi:10.1021/ja0635682

[97] P. S. Dittrich and A. Manz, "Lab-on-a-Chip: Microfluidics in Drug Discovery," Nature Reviews Drug Discovery, Vol. 5, No. 5, 2006, pp. 210-218. doi:10.1038/nrd1985

[98] C. H. Yang, K. S. Huang and J. Y. Chang, "Manufacturing Monodisperse Chitosan Microparticles Containing Ampicillin Using a Microchannel Chip," Biomedical Microdevices, Vol. 9, No. 2, 2007, pp. 253-259. doi:10.1007/s10544-006-9029-Z

[99] C. H. Yang, K. S. Huang, Y. S. Lin, K. Lu, C. C. Tzeng, E. C. Wang, C. H. Lin, W. Y. Hsu and J. Y. Chang, "Microfluidic Assisted Synthesis of Multi-Functional Polycaprolactone Microcapsules: Incorporation of CsTe Quantum Dots, $\mathrm{Fe}_{3} \mathrm{O}_{4}$ Superparamagnetic Nanoparticles and Tamoxifen Anticancer Drugs," Lab Chip, Vol. 9, No. 7, 2009, pp. 961-965. doi:10.1039/b814952f

[100] L. Y. Chu, A. S. Utada, R. K. Shah, J. W. Kim and D. A. Weitz, "Controllable Monodisperse Multiple Emulsion," Angewandte Chemie International Edition, Vol. 46, No. 47, 2007, pp. 8907-8974. doi:10.1002/anie.200702736

[101] G. Karoubi, M. L. Ormiston, D. J. Stewart and D. W. Cour- tman, "Single-Cell Hydrogel Encapsulation for Enhanced Survival of Human Marrow Stromal Cells," Biomaterials, Vol. 30, No. 29, 2009, pp. 5445-5455. doi:10.1016/j.biomaterials.2009.06.035

[102] J. F. Edd, D. Di Carlo, K. J. Humphry, S. Koster, D. Irimia, D. A. Weitz and M. Toner, "Controlled Encapsulation of Single-Cells into Monodisperse Picolitre Drops," Lab Chip, Vol. 8, No. 8, 2008, pp. 1262-1264. doi:10.1039/b805456h

[103] E. Tumarkin, L. Tzadu, E. Csaszar, M. Seo, H. Zhang, A. Lee, R. Peerani, K. Purpura, P. W. Zandstra and E. Kumacheva, "High-Throughput Combinatorial Cell Co-Culture Using Microfluidics," Integrative Biology, Vol. 3, No. 6, 2011, pp. 653-662. doi:10.1039/c1ib00002k

[104] B. M. Leung and M. V. Sefton, "A Modular Approach to Cardiac Tissue Engineering," Tissue Engineering Part A, 2010, Vol. 16, No. 10, pp. 3207-3218. doi:10.1089/ten.tea.2009.0746

[105] D. A. Bruzewicz, A. P. McGuigan and G. M. Whitesides, "Fabrication of a Modular Tissue Construct in a Microfluidic Chip," Lab Chip, Vol. 8, No. 5, 2008, pp. 663-671. doi: $10.1039 / \mathrm{b} 719806 \mathrm{j}$

[106] C. C. Wang, K. C. Yang, K. H. Lin, H. C. Liu and F. H. Lin, "A Highly Organized Three-Dimensional Alginate Scaffold for Cartilage Tissue Engineering Prepared by Microfluidic Technology," Biomaterials, Vol. 32, No. 29, 2011, pp. 7118-7126. doi:10.1016/j.biomaterials.2011.06.018

[107] J. Lee, M. J. Cuddihy, G. M. Cater and N. A. Kotov, "Engineering Liver Tissue Spheroids with Inverted Colloidal Crystal Scaffolds," Biomaterials, Vol. 30, No. 27, 2009, pp. 4687-4694. http://dx.doi:10.1016/j.biomaterials.2009.05.024

[108] C. S. Liu, J. J. Liu, D. Gao M. Y. Ding and J. M. Lin, "Fabrication of Microwell Arrays Based on Two-Dimentional Ordered Polystyrene Microspheres for High-Throughput Single-Cell Analysis," Analytical Chemistry, Vol. 82, No. 22, 2010, pp. 9418-9424. doi:10.1021/ac102094r

[109] C. Y. Li, D. K. Wood, C. M. Hsu and S. N. Bhatia, "DNATemplated Assembly of Droplet-Derived PEG Microtissues," Lab Chip, Vol. 11, No. 17, 2011, pp. 2967-2975. doi:10.1039/c1lc20318e

[110] Y. T. Matsunaga, Y. Morimoto and S. J. Takeuchi, "Molding Cell Beads for Rapid Construction of Macroscopic 3D Tissue Architecture," Advanced Healthcare Materials, Vol. 23, No. 3, 2011, pp. H90-H94.

[111] N. K. Bawolin, M. G. Li, X. B. Chen and W. J. Zhang, "Modeling Material-Degradation-Induced Elastic Property of Tissue Engineering Scaffolds," Journal of Biomechanical Engineering, Vol. 132, No. 11, 2010, pp. 111001-111008. doi: $10.1115 / 1.4002551$ 